\title{
Long time existence of regular solutions to Navier-Stokes equations in cylindrical domains under boundary slip conditions
}

by

\author{
W. M. ZAJĄCZKOWsKI (Warszawa)
}

Abstract. Long time existence of solutions to the Navier-Stokes equations in cylindrical domains under boundary slip conditions is proved. Moreover, the existence of solutions with no restrictions on the magnitude of the initial velocity and the external force is shown. However, we have to assume that the quantity

$$
I=\sum_{i=1}^{2}\left(\left\|\partial_{x_{3}}^{i} v(0)\right\|_{L_{2}(\Omega)}+\left\|\partial_{x_{3}}^{i} f\right\|_{L_{2}(\Omega \times(0, T))}\right)
$$

is sufficiently small, where $x_{3}$ is the coordinate along the axis parallel to the cylinder. The time of existence is inversely proportional to $I$. Existence of solutions is proved by the Leray-Schauder fixed point theorem applied to problems for $h^{(i)}=\partial_{x_{3}}^{i} v, q^{(i)}=\partial_{x_{3}}^{i} p$, $i=1,2$, which follow from the Navier-Stokes equations and corresponding boundary conditions. Existence is proved in Sobolev-Slobodetskiu spaces: $h^{(i)} \in W_{\delta}^{2+\beta, 1+\beta / 2}(\Omega \times$ $(0, T)$ ), where $i=1,2, \beta \in(0,1), \delta \in(1,2), 5 / \delta<3+\beta, 3 / \delta<2+\beta$.

1. Introduction. We consider the following initial-boundary value problem (see $[18,16])$ :

$$
\begin{array}{ll}
v_{, t}+v \cdot \nabla v-\operatorname{div} \mathbb{T}(v, p)=f & \text { in } \Omega^{T}=\Omega \times(0, T), \\
\operatorname{div} v=0 & \text { in } \Omega^{T}, \\
v \cdot \bar{n}=0 & \text { on } S^{T}, \\
\bar{n} \cdot \mathbb{T}(v, p) \cdot \bar{\tau}_{\alpha}=0, \quad \alpha=1,2, & \text { on } S^{T}=S \times(0, T), \\
\left.v\right|_{t=0}=v(0) & \text { in } \Omega,
\end{array}
$$

where $\Omega \subset \mathbb{R}^{3}, S=\partial \Omega, v=v(x, t)=\left(v_{1}(x, t), v_{2}(x, t), v_{3}(x, t)\right) \in \mathbb{R}^{3}$ is the velocity of the fluid motion, $p=p(x, t) \in \mathbb{R}^{1}$ the pressure, $f=f(x, t)=$

2000 Mathematics Subject Classification: 35Q35, 76D03, 76D05.

Key words and phrases: Navier-Stokes equations, motions in cylindrical domains, boundary slip conditions, global existence of regular solutions, large data.

Supported by KBN Grant No. 2 P03A 00223. 
$\left(f_{1}(x, t), f_{2}(x, t), f_{3}(x, t)\right) \in \mathbb{R}^{3}$ the external force field, $\bar{n}$ the unit outward vector normal to the boundary $S$, and $\bar{\tau}_{\alpha}, \alpha=1,2$, are tangent vectors to $S$. Moreover, the dot denotes the scalar product in $\mathbb{R}^{3}$. By $\mathbb{T}(v, p)$ we denote the stress tensor

$$
\mathbb{T}(v, p)=\nu \mathbb{D}(v)-p \mathbb{I},
$$

where $\nu$ is the constant viscosity coefficient, $\mathbb{I}$ the unit matrix and $\mathbb{D}(v)$ the dilatation tensor of the form

$$
\mathbb{D}(v)=\left\{v_{i, x_{j}}+v_{j, x_{i}}\right\}_{i, j=1,2,3} .
$$

Here $\Omega \subset \mathbb{R}^{3}$ is a cylindrical type domain parallel to the $x_{3}$ axis with arbitrary cross-section. We assume that $S_{1}$ is the part of the boundary which is parallel to the $x_{3}$ axis and $S_{2}$ is perpendicular to $x_{3}$. Hence

and

$$
S_{1}=\left\{x \in \mathbb{R}^{3}: \varphi\left(x_{1}, x_{2}\right)=c_{0},-a<x_{3}<a\right\},
$$

$$
S_{2}=\left\{x \in \mathbb{R}^{3}: \varphi\left(x_{1}, x_{2}\right)<c_{0}, x_{3} \text { is equal to either }-a \text { or } a\right\},
$$

where $\varphi\left(x_{1}, x_{2}\right)=c_{0}$ describes a sufficiently smooth closed curve in the plane $x_{3}=$ const and $c_{0}$ is a positive constant.

The aim of this paper is to prove existence of global regular solutions to problem (1.1) without restrictions on the magnitude of $f$ and $v(0)$.

For this purpose we need the existence of weak solutions.

Definition 1.1. By a weak solution to problem (1.1) we mean $v \in$ $V_{2}^{0}\left(\Omega^{T}\right)$ (see Section 2) such that $\operatorname{div} v=0,\left.v \cdot \bar{n}\right|_{S}=0$, satisfying the integral identity

$$
\begin{aligned}
& \int_{\Omega^{T}}\left(-v \cdot \varphi_{, t}+\nu \mathbb{D}(v) \cdot \mathbb{D}(\varphi)+v \cdot \nabla v \cdot \varphi\right) d x d t \\
& \quad+\left.\int_{\Omega} v \cdot \varphi\right|_{t=T} d x-\left.\int_{\Omega} v \cdot \varphi\right|_{t=0} d x=\int_{\Omega^{T}} f \cdot \varphi d x d t
\end{aligned}
$$

for any $\varphi \in W_{2}^{1,1}\left(\Omega^{T}\right)$ such that $\operatorname{div} \varphi=0$ and $\left.\varphi \cdot \bar{n}\right|_{S}=0$.

To prove the existence of weak solutions we need the Korn inequality.

Lemma 1.2 (see also [16, 20]). Assume that

$$
E_{\Omega}(v)=|\mathbb{D}(v)|_{2, \Omega}^{2}<\infty,\left.\quad v \cdot \bar{n}\right|_{S}=0, \quad \operatorname{div} v=0 .
$$

If $\Omega$ is not axially symmetric, then there exists a constant $c$ such that

$$
\|v\|_{1, \Omega}^{2} \leq c E_{\Omega}(v) .
$$

If $\Omega$ is axially symmetric, $\eta=\left(-x_{2}, x_{1}, 0\right), \alpha=\int_{\Omega} v_{\eta} d x$, and $v_{\eta}=v \cdot \eta$, then there exists a constant $c$ such that

$$
\|v\|_{1, \Omega}^{2} \leq c\left(E_{\Omega}(v)+\left|\int_{\Omega} v_{\eta} d x\right|^{2}\right),
$$

where the notation is described in Section 2. 
Proof. First, consider nonaxially symmetric domains. We have

$$
E_{\Omega}(v)=\int_{\Omega}\left(v_{i, x_{j}}+v_{j, x_{i}}\right)^{2} d x=2\left(\int_{\Omega} v_{i, x_{j}}^{2} d x+\int_{\Omega} v_{i, x_{j}} v_{j, x_{i}} d x\right),
$$

where the summation convention over repeated indices is assumed. After integrating by parts and applying $(1.1)_{2,3}$, the second integral on the r.h.s. of (1.8) equals $-\int_{S} n_{i, x_{j}} v_{i} v_{j} d S$. Using this in (1.8) yields

$$
|\nabla v|_{2, \Omega}^{2} \leq c\left(E_{\Omega}(v)+|v|_{2, S}^{2}\right) \text {. }
$$

By the trace theorem we have

$$
|\nabla v|_{2, \Omega}^{2} \leq c\left(E_{\Omega}(v)+|v|_{2, \Omega}^{2}\right) .
$$

Making use of the fact that $E_{\Omega}(u)=0,\left.u \cdot \bar{n}\right|_{S}=0$ implies $u=0$ and repeating the proof of Lemma 3.2 from [20], we obtain

$$
|v|_{2, \Omega}^{2} \leq \delta|\nabla v|_{2, \Omega}^{2}+M E_{\Omega}(v),
$$

where $\delta$ can be chosen as small as we need and $M=M(\delta)$ is some constant. In view of (1.10) and (1.11) inequality (1.6) follows.

Assume now that $\Omega$ is axially symmetric. Then we decompose $v$ as

$$
v=v^{\prime}+\frac{\alpha}{\int_{\Omega} \eta^{2} d x} \eta
$$

where $\int_{\Omega} v^{\prime} \cdot \eta d x=0$. Then, instead of (1.11), we have

$$
\left|v^{\prime}\right|_{2, \Omega}^{2} \leq \delta\left|\nabla v^{\prime}\right|_{2, \Omega}^{2}+M E_{\Omega}\left(v^{\prime}\right) .
$$

Using the fact that $E_{\Omega}(v)=E_{\Omega}\left(v^{\prime}\right)$ and

$$
\left|\nabla v^{\prime}\right|_{2, \Omega} \leq c|\nabla v|_{2, \Omega}+c\left|\int_{\Omega} v_{\eta} d x\right|
$$

we obtain from (1.13) the inequality

$$
|v|_{2, \Omega}^{2} \leq \delta|\nabla v|_{2, \Omega}^{2}+M E_{\Omega}(v)+c\left|\int_{\Omega} v_{\eta} d x\right|^{2} .
$$

Employing (1.14) in (1.10) yields (1.7), which ends the proof.

Now we obtain energy type estimates for solutions of (1.1).

Lemma 1.3. Assume $f \in L_{\infty}\left(0, \infty ; L_{6 / 5}(\Omega)\right), \int_{\Omega^{t}} f_{\eta} d x d t^{\prime} \in L_{\infty}(0, \infty)$ and $v(0) \in L_{2}(\Omega)$. Let $T>0$ be given. Assume that there exist constants $a_{1}, a_{2}$ such that

$$
a_{1} \equiv \sup _{t}|f(t)|_{6 / 5, \Omega}<\infty, \quad a_{2} \equiv \sup _{t}\left|\int_{\Omega^{t}} f_{\eta} d x d t^{\prime}\right|<\infty .
$$


Then there exist constants

$$
\begin{aligned}
& d_{1}^{2}=\frac{c}{\nu_{1}} a_{1}^{2}+|v(0)|_{2, \Omega}^{2}, \\
& d_{2}^{2}=\left(\min \left(1, \nu_{2}\right)\right)^{-1} e^{\nu_{1} T}\left(\frac{c}{\nu_{1}} a_{1}^{2}+d_{1}^{2}\right), \\
& d_{3}^{2}=\frac{c}{\nu_{1}}\left(a_{1}^{2}+a_{2}^{2}+\left|\int_{\Omega} v_{\eta}(0) d x\right|^{2}\right)+|v(0)|_{2, \Omega}^{2}, \\
& d_{4}^{2}=\left(\min \left(1, \nu_{2}\right)\right)^{-1} e^{\nu_{1} T}\left[\frac{c}{\nu_{1}}\left(a_{1}^{2}+a_{2}^{2}+\mid \int_{\Omega} v_{\eta}\left(\left.0 d x\right|^{2}\right)+d_{3}^{2}\right],\right.
\end{aligned}
$$

which do not depend on $k_{0}=k T, k \in \mathbb{N}$, and $\nu / c=\nu_{1}+\nu_{2}$, where $c$ is the constant either from (1.6) or from (1.7), such that in the nonaxially symmetric case we have

$$
\begin{array}{ll}
|v(t)|_{2, \Omega} \leq d_{1} & \text { for any } t \geq 0, \\
\|v\|_{V_{2}^{0}(\Omega \times(k T, t))} \leq d_{2} & \text { for } t \in(k T,(k+1) T), k \in \mathbb{N},
\end{array}
$$

and in the axially symmetric case

$$
\begin{array}{ll}
|v(t)|_{2, \Omega} \leq d_{3} & \text { for any } t \geq 0, \\
\|v\|_{V_{2}^{0}(\Omega \times(k T, t))} \leq d_{4} & \text { for } t \in(k T,(k+1) T), k \in \mathbb{N} .
\end{array}
$$

Proof. First we consider the nonaxially symmetric case. Multiplying $(1.1)_{1}$ by $v$ and integrating over $\Omega$ we obtain

$$
\frac{1}{2} \frac{d}{d t}|v|_{2, \Omega}^{2}+\nu E_{\Omega}(v)=\int_{\Omega} f \cdot v d x .
$$

Applying (1.6) yields

$$
\frac{1}{2} \frac{d}{d t}|v|_{2, \Omega}^{2}+\frac{\nu}{c}\|v\|_{1, \Omega}^{2} \leq \int_{\Omega} f \cdot v d x .
$$

Hence we have

$$
\frac{d}{d t}|v|_{2, \Omega}^{2}+\frac{\nu}{c}\|v\|_{1, \Omega}^{2} \leq c|f|_{6 / 5, \Omega}^{2} .
$$

Let $\nu / c=\nu_{1}+\nu_{2}$. Then (1.21) takes the form

$$
\frac{d}{d t}|v|_{2, \Omega}^{2}+\nu_{1}|v|_{2, \Omega}^{2}+\nu_{2}\|v\|_{1, \Omega}^{2} \leq c|f|_{6 / 5, \Omega}^{2} .
$$

This implies

$$
\frac{d}{d t}\left(|v|_{2, \Omega}^{2} e^{\nu_{1} t}\right)+\nu_{2}\|v\|_{1, \Omega}^{2} e^{\nu_{1} t} \leq c|f|_{6 / 5, \Omega}^{2} e^{\nu_{1} t} .
$$

Integrating (1.23) with respect to time yields 


$$
\begin{aligned}
|v(t)|_{2, \Omega}^{2}+\nu_{2} e^{-\nu_{1} t} & \int_{0}^{t}\left\|v\left(t^{\prime}\right)\right\|_{1, \Omega}^{2} e^{\nu_{1} t^{\prime}} d t^{\prime} \\
\leq & c e^{-\nu_{1} t} \int_{0}^{t}\left|f\left(t^{\prime}\right)\right|_{6 / 5, \Omega}^{2} e^{\nu_{1} t^{\prime}} d t^{\prime}+e^{-\nu_{1} t}|v(0)|_{2, \Omega}^{2} .
\end{aligned}
$$

Considering (1.24) for any $t>0$ we omit the second term on the l.h.s. because it is bounded from below by $e^{-\nu_{1} t} \int_{0}^{t}\left\|v\left(t^{\prime}\right)\right\|_{1, \Omega}^{2} d t^{\prime}$, which does not imply any estimate for $\int_{0}^{t}\left\|v\left(t^{\prime}\right)\right\|_{1, \Omega}^{2} d t^{\prime}$ for any $t \in \mathbb{R}_{+}$. Then by the assumptions of the lemma we have

$$
|v(t)|_{2, \Omega}^{2} \leq \frac{c}{\nu_{1}} a_{1}^{2}+e^{-\nu_{1} t}|v(0)|_{2, \Omega}^{2} \leq d_{1}^{2}
$$

which implies $(1.17)_{1}$.

Having estimate (1.25) for any $t>0$ we can consider (1.24) in the interval $(k T,(k+1) T), k \in \mathbb{N}$. Hence for $t \in(k T,(k+1) T)$ we have

$$
\begin{aligned}
|v(t)|_{2, \Omega}^{2}+\nu_{2} e^{-\nu_{1} t} & \int_{k T}^{t}\left\|v\left(t^{\prime}\right)\right\|_{1, \Omega}^{2} e^{\nu_{1} t^{\prime}} d t^{\prime} \\
\leq & c a_{1}^{2} e^{-\nu_{1} t} \int_{k T}^{t} e^{\nu_{1} t^{\prime}} d t^{\prime}+e^{-\nu_{1}(t-k T)}|v(k T)|_{2, \Omega}^{2} .
\end{aligned}
$$

Continuing,

$$
\begin{aligned}
|v(t)|_{2, \Omega}^{2}+\nu_{2} e^{-\nu_{1}(t-k T)} \int_{k T}^{t} \| v\left(t^{\prime}\right) & \|_{1, \Omega}^{2} d t^{\prime} \\
& \leq \frac{c}{\nu_{1}} a_{1}^{2}+e^{-\nu_{1}(t-k T)}|v(k T)|_{2, \Omega}^{2}
\end{aligned}
$$

Finally, employing (1.25) in (1.27) yields

$$
|v(t)|_{2, \Omega}^{2}+\nu_{2} e^{-\nu_{1} T} \int_{k T}^{t}\left\|v\left(t^{\prime}\right)\right\|_{1, \Omega}^{2} d t^{\prime} \leq \frac{c}{\nu_{1}} a_{1}^{2}+d_{1}^{2},
$$

which implies

$$
|v(t)|_{2, \Omega}^{2}+\nu_{2} \int_{k T}^{t}\left\|v\left(t^{\prime}\right)\right\|_{1, \Omega}^{2} d t^{\prime} \leq e^{\nu_{1} T}\left(\frac{c}{\nu_{1}} a_{1}^{2}+d_{1}^{2}\right),
$$

where $t \in(k T,(k+1) T), k \in \mathbb{N}$. Hence (1.28) implies $(1.17)_{2}$.

Now we consider the axially symmetric case. In view of (1.7) instead of (1.22) we have

$$
\frac{d}{d t}|v|_{2, \Omega}^{2}+\nu_{1}|v|_{2, \Omega}^{2}+\nu_{2}\|v\|_{1, \Omega}^{2} \leq c|f|_{6 / 5, \Omega}^{2}+\left|\int_{\Omega} v_{\eta} d x\right|^{2} .
$$


To examine the last integral on the r.h.s., we multiply $(1.1)_{1}$ by $\eta$ and integrate over $\Omega^{t}$. Using the fact that $\left.\eta \cdot \bar{n}\right|_{S}=0,(1.1)_{3.4}$ and the fact that $\nabla \eta$ is an antisymmetric tensor we obtain

$$
\int_{\Omega} v_{\eta} d x=\int_{\Omega^{t}} f_{\eta} d x d t^{\prime}+\int_{\Omega} v_{\eta}(0) d x .
$$

Inserting (1.30) in (1.29) yields

$$
\begin{aligned}
\frac{d}{d t}|v|_{2, \Omega}^{2}+\nu_{1}|v|_{2, \Omega}^{2} & +\nu_{2}\|v\|_{1, \Omega}^{2} \\
& \leq c|f|_{6 / 5, \Omega}^{2}+c\left|\int_{\Omega^{t}} f_{\eta} d x d t^{\prime}\right|^{2}+c\left|\int_{\Omega} v_{\eta}(0) d x\right|^{2} .
\end{aligned}
$$

Repeating the considerations leading to (1.24) we obtain

$$
\begin{aligned}
& |v(t)|_{2, \Omega}^{2}+\nu_{2} e^{-\nu_{1} t} \int_{0}^{t}\left\|v\left(t^{\prime}\right)\right\|_{1, \Omega}^{2} e^{\nu_{1} t^{\prime}} d t^{\prime} \\
& \leq c e^{-\nu_{1} t} \int_{0}^{t}\left|f\left(t^{\prime}\right)\right|_{6 / 5, \Omega}^{2} e^{\nu_{1} t^{\prime}} d t^{\prime}+c e^{-\nu_{1} t} \int_{0}^{t}\left|\int_{\Omega^{t^{\prime}}} f_{\eta} d x d t^{\prime \prime}\right|^{2} e^{\nu_{1} t^{\prime}} d t^{\prime} \\
& \quad+c e^{-\nu_{1} t} \int_{0}^{t}\left|\int_{\Omega} v_{\eta}(0) d x\right|^{2} e^{\nu_{1} t^{\prime}} d t^{\prime}+e^{-\nu_{1} t}|v(0)|_{2, \Omega}^{2} .
\end{aligned}
$$

Applying (1.15) we have

$$
\begin{aligned}
|v(t)|_{2, \Omega}^{2}+\nu_{2} e^{-\nu_{1} t} & \int_{0}^{t}\left\|v\left(t^{\prime}\right)\right\|_{1, \Omega}^{2} e^{\nu_{1} t^{\prime}} d t^{\prime} \\
\leq & \frac{c}{\nu_{1}}\left(a_{1}^{2}+a_{2}^{2}+\left|\int_{\Omega} v_{\eta}(0) d x\right|^{2}\right)+e^{-\nu_{1} t}|v(0)|_{2, \Omega}^{2}
\end{aligned}
$$

Hence

$$
|v(t)|_{2, \Omega}^{2} \leq \frac{c}{\nu_{1}}\left(a_{1}^{2}+a_{2}^{2}+\left|\int_{\Omega} v_{\eta}(0) d x\right|^{2}\right)+e^{-\nu_{1} t}|v(0)|_{2, \Omega}^{2} \leq d_{3}^{2},
$$

which implies $(1.18)_{1}$.

Having estimate (1.34) we can consider (1.33) in the interval $(k T,(k+1) T)$, $k \in \mathbb{N}$,

$$
\begin{aligned}
|v(t)|_{2, \Omega}^{2} & +\nu_{2} e^{-\nu_{1} t} \int_{k T}^{t}\left\|v\left(t^{\prime}\right)\right\|_{1, \Omega}^{2} e^{\nu_{1} t^{\prime}} d t^{\prime} \\
\leq & \frac{c}{\nu_{1}}\left(a_{1}^{2}+a_{2}^{2}+\left|\int_{\Omega} v_{\eta}(0) d x\right|^{2}\right)+e^{-\nu_{1}(t-k T)}|v(k T)|_{2, \Omega}^{2},
\end{aligned}
$$


where $t \in(k T,(k+1) T)$. In view of (1.34) we obtain from (1.35) the inequality

$$
\begin{aligned}
|v(t)|_{2, \Omega}^{2}+\nu_{2} e^{-\nu_{1} T} \int_{k T}^{t}\left\|v\left(t^{\prime}\right)\right\|_{1, \Omega}^{2} d t^{\prime} & \\
& \leq \frac{c}{\nu_{1}}\left(a_{1}^{2}+a_{2}^{2}+\left|\int_{\Omega} v_{\eta}(0) d x\right|^{2}\right)+d_{3}^{2},
\end{aligned}
$$

which implies $(1.18)_{2}$. This ends the proof.

From Lemma 1.3 by an application of the Galerkin method and the considerations from $[9$, Ch. 6] we have

Lemma 1.4. Let the assumptions of Lemma 1.3 hold. Then there exists a weak solution to problem (1.1) in any interval $(k T,(k+1) T), k \in \mathbb{N}$, satisfying

$$
\|v\|_{V_{2}^{0}(\Omega \times(k T,(k+1) T)} \leq d_{i}
$$

where $i=2$ for nonaxially symmetric domains and $i=4$ for axially symmetric domains.

The aim of this paper is to prove global existence of regular solutions to problem (1.1) by improving regularity of the weak solution. For this purpose we follow the ideas from $[18,19,20]$. Since in this paper nonaxially symmetric domains are considered, we do not use the cylindrical coordinates $r, \varphi, z$. Compared to $[18,19]$ we replace the cylindrical coordinate $\varphi$ by $x_{3}$; $v_{\varphi}$ by $v_{3} ; h=v_{r, \varphi} \bar{e}_{r}+v_{\varphi, \varphi} \bar{e}_{\varphi}+v_{z, \varphi} \bar{e}_{z}$ by $h^{(1)}=v_{, x_{3}} ;$ and $\chi=(\operatorname{rot} v)_{\varphi}$ by $\chi=\operatorname{rot} v \cdot \bar{e}_{3}$. Here $\bar{e}_{r}=(\cos \varphi, \sin \varphi, 0), \bar{e}_{\varphi}=(-\sin \varphi, \cos \varphi, 0), \bar{e}_{z}=$ $(0,0,1)=\bar{e}_{3}, v_{r}=v \cdot \bar{e}_{r}, v_{\varphi}=v \cdot \bar{e}_{\varphi}, v_{z}=v \cdot \bar{e}_{z}$.

Hence we introduce the quantities

$$
\begin{aligned}
& h^{(1)}=v_{, x_{3}}, \quad q^{(1)}=p_{, x_{3}}, \quad h^{(2)}=v_{, x_{3} x_{3}}, \quad q^{(2)}=p_{, x_{3} x_{3}}, \\
& w=v_{3}, \quad \chi=v_{2, x_{1}}-v_{1, x_{2}} .
\end{aligned}
$$

Now we formulate the main results of this paper. First we introduce the quantities

$$
\begin{aligned}
G_{1}(T)= & \|f\|_{L_{\infty}\left(0, T ; L_{6 / 5}(\Omega)\right)}+\|f\|_{L_{27 / 16}\left(\Omega^{T}\right)}+\left\|(\operatorname{rot} f)_{3}\right\|_{L_{18 / 13}\left(\Omega^{T}\right)} \\
& +\left\|(\operatorname{rot} f)^{\prime}\right\|_{L_{2}\left(S_{2}^{T}\right)}+\left\|f_{, x_{3}}\right\|_{L_{2}\left(\Omega^{T}\right)}+\left\|f_{, x_{3} x_{3}}\right\|_{L_{2}\left(\Omega^{T}\right)}, \\
G_{0}(0)= & \|v(0)\|_{W_{27 / 16}^{22 / 27}(\Omega)}+\left\|(\operatorname{rot} v(0))_{3}\right\|_{L_{2}(\Omega)}+\left\|v_{x_{3}}(0)\right\|_{L_{2}(\Omega)} \\
& +\left\|v_{, x_{3} x_{3}}(0)\right\|_{L_{2}(\Omega)}, \\
d(T)= & \sum_{i=1}^{2}\left(\left\|\partial_{x_{3}}^{i} f\right\|_{L_{2}\left(\Omega^{T}\right)}+\left\|\partial_{x_{3}}^{i} v(0)\right\|_{L_{2}(\Omega)}\right) \\
& +\left\|(\operatorname{rot} f)^{\prime}\right\|_{L_{2}\left(S_{2}^{T}\right)}+\left|f_{3}\right|_{L_{2}\left(S_{2}^{T}\right)},
\end{aligned}
$$




$$
\begin{aligned}
D(T)= & \sum_{i=1}^{2}\left(\left\|\partial_{x_{3}}^{i} f\right\|_{W_{\delta}^{\beta, \beta / 2}\left(\Omega^{T}\right)}+\left\|\partial_{x_{3}}^{i} v(0)\right\|_{W_{\delta}^{2+\beta-2 / \delta}(\Omega)}\right) \\
& +\left\|(\operatorname{rot} f)^{\prime}\right\|_{W_{\delta}^{1+\beta-1 / \delta, 1 / 2+1 / 2 \beta-1 / 2 \delta}\left(S_{2}^{T}\right)}
\end{aligned}
$$

(here $(\operatorname{rot} f)^{\prime}=\left((\operatorname{rot} f)_{1},(\operatorname{rot} f)_{2}\right)$, where $(\operatorname{rot} f)_{i}$ is the $i$ th Cartesian coordinate of $\operatorname{rot} f)$, and

$$
\gamma(T)=\sum_{i=1}^{2}\left(\left\|\partial_{x_{3}}^{i} v\right\|_{W_{\delta}^{2+\beta, 1+\beta / 2}\left(\Omega^{T}\right)}+\left\|\nabla \partial_{x_{3}}^{i} p\right\|_{W_{\delta}^{\beta, \beta / 2}\left(\Omega^{T}\right)}\right) .
$$

Finally, for $\beta \geq 0$ and $\delta \in[1, \infty]$ we define

$$
\begin{aligned}
W_{\delta}^{\beta, \beta / 2}\left(\Omega^{T}\right)= & \left\{u:\|u\|_{W_{\delta}^{\beta, \beta / 2}\left(\Omega^{T}\right)} \equiv\|u\|_{L_{\delta}\left(\Omega^{T}\right)}\right. \\
& +\left(\int_{0}^{T} \int_{\Omega} \int_{\Omega} \frac{\left|D_{x}^{[\beta]} u(x, t)-D_{x^{\prime}}^{[\beta]} u\left(x^{\prime}, t\right)\right|^{\delta}}{\left|x-x^{\prime}\right|^{3+\delta(\beta-[\beta])}} d x d x^{\prime} d t\right)^{1 / \delta} \\
& \left.+\left(\int_{\Omega}^{T} \int_{0}^{T} \int_{0}^{T} \frac{\left|\partial_{t}^{[\beta / 2]} u(x, t)-\partial_{t^{\prime}}^{[\beta / 2]} u\left(x, t^{\prime}\right)\right|^{\delta}}{\left|t-t^{\prime}\right|^{1+\delta(\beta / 2-[\beta / 2])}} d x d t d t^{\prime}\right)^{1 / \delta}<\infty\right\} .
\end{aligned}
$$

where $[\beta]$ is the integer part of $\beta$.

TheOREM 1 (local existence). Assume that $\Omega$ is a cylindrical type domain with respect to the $x_{3}$ axis and with an arbitrary cross-section. Its boundary is composed of two parts: $S_{1}$, parallel to $x_{3}$, and $S_{2}$, perpendicular to $x_{3} ; S_{1} \in C^{3}$. Assume $v(0) \in W_{27 / 16}^{22 / 27}(\Omega),(\operatorname{rot} v(0))_{3} \in L_{2}(\Omega), v_{, x_{3}}(0) \in$ $W_{\delta}^{2+\beta-2 / \delta}(\Omega), v_{, x_{3} x_{3}}(0) \in W_{\delta}^{2+\beta-2 / \delta}(\Omega), f \in L_{6 / 5, \infty}\left(\Omega^{T}\right) \cap L_{27 / 16}\left(\Omega^{T}\right)$, $(\operatorname{rot} f)_{3} \in L_{18 / 13}\left(\Omega^{T}\right),\left.(\operatorname{rot} f)^{\prime}\right|_{S_{2}} \in W_{\delta}^{1+\beta-1 / \delta, 1 / 2-\beta / 2-1 /(2 \delta)}\left(S_{2}^{T}\right), f_{3} \in$ $L_{2}\left(S_{2}^{T}\right), f_{, x_{3}}, f_{, x_{3} x_{3}} \in W_{\delta}^{\beta, \beta / 2}\left(\Omega^{T}\right), \delta \in(1,2), \beta \in(0,1), 5 / \delta<3+\beta$, $3 / \delta<2+\beta$. Then for sufficiently small $d(T)$ there exists a solution to problem (1.1) such that $\partial_{x_{3}}^{i} v \in W_{\delta}^{2+\beta, 1+\beta / 2}\left(\Omega^{T}\right), \partial_{x_{3}}^{i} \nabla p \in W_{\delta}^{\beta, \beta / 2}\left(\Omega^{T}\right)$, $i=1,2$.

Moreover, there exist a function $\varphi=\varphi\left(\gamma(T), G_{1}(T), G_{0}(0), T\right)$ and $a$ constant $c_{1}$ with the following properties (see Lemma 5.2). Assume that for a given $T$ there exists a constant $A$ such that

$$
\varphi\left(A, G_{1}(T), G_{0}(0), T\right) d(T)+c_{1} D(T) \leq A
$$

and

$$
c_{1} D(T)<A
$$

Then the local solution is such that

$$
\gamma(T) \leq A
$$


Finally, (1.39) implies

$$
T<\frac{1}{\varphi_{3}(d(T))}
$$

with $\lim _{d \rightarrow 0} \varphi_{3}(d)=0$.

Hence, we have existence for arbitrary $T$ if $d(T)$ is sufficiently small.

Theorem 2 (uniqueness). Solutions to problem (1.1) such that $v \in$ $L_{2}\left(0, T ; W_{3}^{1}(\Omega)\right)$ are unique.

To prove Theorem 1 we use problems (3.1) and (3.12) for $\left(h^{(i)}, q^{(i)}\right)$, $i=1,2$, respectively, in the form (5.1) and (5.2) which is appropriate for applying the Leray-Schauder fixed point theorem. For this purpose we need to estimate $v$ in terms of $h^{(1)}$ and $h^{(2)}$. The relation between $v$ and $h^{(1)}$, $h^{(2)}$ and the corresponding estimate (4.28) for $v \in W_{27 / 16}^{2,1}\left(\Omega^{T}\right)$ is described in Lemma 4.4. To obtain (4.28) we use anisotropic energy type estimates described by Lemma 2.1, which implies a high-regularity estimate for $v$ with different smoothness with respect to $x^{\prime}=\left(x_{1}, x_{2}\right)$ and $x_{3}$ (see Lemma 4.3).

The fact that $v \in W_{27 / 16}^{2,1}\left(\Omega^{T}\right)$ is crucial because $27 / 16>5 / 3$ and $v \in W_{5 / 3}^{2,1}\left(\Omega^{T}\right)$ implies that $v \cdot \nabla v \in L_{5 / 3}\left(\Omega^{T}\right)$ so (1.1) does not increase regularity. Moreover, for $v \in W_{r}^{2,1}\left(\Omega^{T}\right), r>5 / 3$, interpolation inequalities from the proofs of Lemmas 4.6 and 4.7 are such that the mapping $\Phi$ is compact (see Lemma 5.1) and estimate (5.9) holds (see Lemma 5.2).

The result of Theorem 1 is a small step towards solving the regularity problem for the Navier-Stokes equations, because existence of a regular solution with small variations in the direction of the axis of a cylindrical domain is proved. It seems that the solution is close to two-dimensional (see [10]). But in [10] the non-slip condition is imposed on the boundary. Hence the solution in Theorem 1 would be close to a two-dimensional solution with slip boundary condition. Now, we describe the differences between the latter solution and the solution presented in the present paper. In the $2 \mathrm{~d}$-solution the boundary conditions on $S_{2}$ are satisfied automatically, while in this paper they are not. Moreover, in the two cases we use different estimates and different imbedding theorems. In the 3d-case the imbedding theorems and interpolation inequalities applied are much weaker than in the $2 \mathrm{~d}$-case.

Moreover, in the $2 \mathrm{~d}$-case global estimate follows directly from imbedding theorems (see [10]), whereas in the present paper, much work is necessary to obtain an estimate guaranteeing global existence (see Lemma 5.2).

In the last decade a lot of papers concerning global regular special $3 \mathrm{~d}-$ solutions to Navier-Stokes equations have appeared (see $[2,3,7,10,13$, 14, 15, 18-20]). They base on lower-dimensional global regular solutions to Navier-Stokes equations: 
1. two-dimensional [10];

2. axially-symmetric $[11,17]$;

3. helically symmetric [12].

Global regular solutions close to $(1.43)_{1}$ are shown in this paper and also for thin domains in $[2,3,7,13-15]$.

In [13-15] Raugel and Sell proved existence of global regular solutions to the Navier-Stokes equations in a thin domain $\Omega_{\varepsilon}=\Omega^{\prime} \times(0, \varepsilon), \Omega^{\prime} \subset \mathbb{R}^{2}$, $\varepsilon$ small, with periodic boundary conditions by using the semigroup technique. The result was generalized by Avrin [2,3], who also proved existence of global regular solutions in the thin domain $\Omega_{\varepsilon}$ but with Dirichlet boundary conditions on $\partial \Omega^{\prime}$ and periodic conditions in the third direction. In his considerations the smallness of $\varepsilon$ was replaced by large first eigenvalue of $-P \Delta$, where $P$ is the projection operator on the divergence free vector fields. To prove this he used a fixed point argument. The above results were generalized by Iftimie and Raugel in [7] who relaxed the conditions on the magnitude and regularity of $v(0)$ and $f$.

Global regular solutions close to $(1.43)_{2}$ have been found by the author in $[18-21]$.

Finally, in $[4,5]$ there are some generalizations of solutions from $(1.43)_{3}$.

2. Notation and auxiliary results. To simplify the writing we introduce the following notation:

$$
\begin{array}{ll}
|u|_{p, Q}=\|u\|_{L_{p}(Q)}, & Q \in\left\{\Omega^{T}, S^{T}, \Omega, S\right\}, p \in[1, \infty], \\
\|u\|_{s, Q}=\|u\|_{H^{s}(Q)}, & Q \in\{\Omega, S\}, s \in \mathbb{R}_{+} \cup\{0\}, \\
\|u\|_{s, Q^{T}}=\|u\|_{W_{2}^{s, s / 2}\left(Q^{T}\right)}, & Q \in\{\Omega, S\}, s \in \mathbb{R}_{+} \cup\{0\}, \\
|u|_{p, q, Q^{T}}=\|u\|_{L_{q}\left(0, T ; L_{p}(Q)\right)}, & Q \in\{\Omega, S\}, p, q \in[1, \infty], \\
\|u\|_{s, q, Q^{T}}=\|u\|_{W_{q}^{s, s / 2}\left(Q^{T}\right)}, & Q \in\{\Omega, S\}, s \in \mathbb{R}_{+} \cup\{0\}, q \in[1, \infty] . \\
\|u\|_{s, q, Q}=\|u\|_{W_{q}^{s}(Q)}, & Q \in\{\Omega, S\}, s \in \mathbb{R}_{+} \cup\{0\}, q \in[1, \infty] .
\end{array}
$$

By $c$ we denote a generic constant which may change its value from formula to formula. By $c(\sigma), c_{k}(\sigma), k \in \mathbb{N}, \varphi(\sigma)$ we understand generic functions which are always positive and increasing. We do not distinguish the scalar and vector-valued functions.

Moreover, we use the abbreviation r.h.s. (l.h.s) for right-hand side (lefthand side). 
We introduce the space

$$
\begin{aligned}
V_{2}^{k}\left(\Omega^{T}\right)=\left\{u:\|u\|_{V_{2}^{k}\left(\Omega^{T}\right)}=\right. & \operatorname{ess}_{t \in(0, T)}\|u\|_{H^{k}(\Omega)} \\
& \left.+\left(\int_{0}^{T}\|\nabla u\|_{H^{k}(\Omega)} d t\right)^{1 / 2}<\infty\right\}, \quad k \in \mathbb{N} .
\end{aligned}
$$

Now we recall an imbedding for anisotropic Sobolev spaces. Let $\Omega \subset \mathbb{R}^{3}$. Then we define

$$
\|u\|_{W_{2}^{1, k}(\Omega)}=\left[\int_{\Omega}\left(|u|^{2}+\left|\nabla^{\prime} u\right|^{2}+\left|\nabla_{x_{3}}^{k} u\right|^{2}\right) d x\right]^{1 / 2}, \quad k \in \mathbb{N},
$$

where $\nabla^{\prime}=\left(\partial_{x_{1}}, \partial_{x_{2}}\right)$.

LEMmA 2.1. Assume that $u \in L_{\infty}\left(0, T ; L_{2}(\Omega)\right) \cap L_{2}\left(0, T ; W_{2}^{1, k}(\Omega)\right), 1 \leq$ $k \in \mathbb{N}$. Then $u \in L_{r}\left(0, T ; L_{q}(\Omega)\right)$ for any $r, q \geq 1$ satisfying

$$
\frac{2}{r}+\frac{2 k+1}{q k}=\frac{2 k+1}{2 k} \text {, }
$$

and

$$
|u|_{q, r, \Omega^{T}} \leq c\left(\operatorname{ess~sup}_{t \in[0, T]}|u|_{2, \Omega}+\|u\|_{L_{2}\left(0, T ; W_{2}^{1, k}(\Omega)\right)}\right) .
$$

In the case $r=q$ we have

$$
q=\frac{2(4 k+1)}{2 k+1} \equiv q(k),
$$

so that $q(1)=\frac{10}{3}, q(2)=\frac{18}{3}, q(3)=\frac{26}{7}, \ldots$, and $\lim _{k \rightarrow \infty} q(k)=4$.

Proof. From [6, Ch. 3, Sect. 10] for $u \in W_{2}^{1, k}(\Omega), q \geq 1$ and any $\varepsilon>0$ we have the inequality

$$
|u|_{q, \Omega} \leq \varepsilon^{1-\varkappa}\|u\|_{W_{2}^{1, k}(\Omega)}+c \varepsilon^{-\varkappa}|u|_{2, \Omega},
$$

provided

$$
\varkappa=1-\frac{2}{q}+\left(\frac{1}{2}-\frac{1}{q}\right) \frac{1}{k}<1
$$

which holds if

$$
q<4 k+2
$$

Inserting in $(2.4) \varepsilon=\epsilon^{\frac{1}{\varkappa(1-\varkappa)}}$ yields

$$
|u|_{q, \Omega} \leq \epsilon^{1 / \varkappa}\|u\|_{W_{2}^{1, k}(\Omega)}+c \epsilon^{-1 /(1-\varkappa)}|u|_{2, \Omega} .
$$

Equalizing the terms on the r.h.s. of (2.7) gives

$$
\epsilon^{1 / \varkappa}\|u\|_{W_{2}^{1, k}(\Omega)}=\epsilon^{-1 /(1-\varkappa)}|u|_{2, \Omega},
$$


which implies

$$
\epsilon=\left(\frac{|u|_{2, \Omega}}{\|u\|_{W_{2}^{1, k}(\Omega)}}\right)^{\varkappa(1-\varkappa)} .
$$

Inserting (2.8) in (2.7) yields

$$
|u|_{q, \Omega} \leq c\|u\|_{W_{2}^{1, k}(\Omega)}^{\varkappa}|u|_{2, \Omega}^{1-\varkappa}
$$

provided (2.6) holds and

$$
\varkappa=\frac{2 k+1}{2 k}-\frac{2 k+1}{q k}<1 .
$$

Next we calculate

$$
\begin{aligned}
|u|_{q, r, \Omega^{T}} & =\left(\int_{0}^{T}|u(t)|_{q, \Omega}^{r} d t\right)^{1 / r} \\
& \leq c\left(\int_{0}^{T}\|u(t)\|_{W_{2}^{1, k}(\Omega)}^{\varkappa r} d t\right)^{1 / r} \underset{t}{\operatorname{ess} \sup }|u|_{2, \Omega}^{1-\varkappa}
\end{aligned}
$$

for any $\varkappa \in(0,1)$. Putting

$$
\varkappa=\frac{2}{r}
$$

we obtain

$$
|u|_{q, r, \Omega^{T}} \leq c\|u\|_{L_{2}\left(0, T ; W_{2}^{1, k}(\Omega)\right)}^{2 / r} \operatorname{ess} \sup _{t}|u|_{2, \Omega}^{1-2 / r} .
$$

By the Young inequality, (2.13) implies

$$
|u|_{q, r, \Omega^{T}} \leq \varepsilon^{r / 2}\|u\|_{L_{2}\left(0, T ; W_{2}^{1, k}(\Omega)\right)}+c \varepsilon^{-r /(r-2)} \underset{t}{\operatorname{ess} \sup }|u|_{2, \Omega}
$$

for all $\varepsilon \in \mathbb{R}_{+}$. From (2.10), (2.12) and (2.13) we get (2.1), (2.2), which ends the proof.

For $q=r$ inequality (2.14) takes the form

$$
|u|_{q, \Omega^{T}} \leq \varepsilon^{q / 2}\|u\|_{L_{2}\left(0, T ; W_{2}^{1, k}(\Omega)\right)}+c \varepsilon^{-q /(q-2)} \operatorname{ess} \sup _{t}|u|_{2, \Omega},
$$

where $q$ is defined by (2.3).

In this paper we use frequently the imbedding inequality

$$
\left|\nabla^{i} u\right|_{p, q, \Omega^{T}} \leq c\|u\|_{2+\beta, \delta, \Omega^{T}}, \quad i \leq 2,
$$

which holds for

$$
\frac{5}{\delta}-\frac{3}{p}-\frac{2}{q}+i \leq 2+\beta .
$$

In the case when either $p$ or $q$ is equal to $\infty$, the inequality in (2.17) must be strict. 
Now we formulate the results of [1], which are frequently used in this paper. Let us consider the Stokes problem

$$
\begin{array}{ll}
v_{, t}-\operatorname{div} \mathbb{T}(v, p)=f & \text { in } \Omega^{T}, \\
\operatorname{div} v=g & \text { in } \Omega^{T}, \\
\bar{n} \cdot \mathbb{D}(v) \cdot \bar{\tau}_{i}=b_{i}, \quad i=1,2, & \text { on } S^{T}, \\
v \cdot \bar{n}=b_{3} & \text { on } S^{T}, \\
\left.v\right|_{t=0}=v(0) & \text { in } \Omega .
\end{array}
$$

Let us introduce the Besov spaces:

$$
\begin{aligned}
B_{r, q}^{2 s, s}\left(\Omega^{T}\right)=\left\{u:\|u\|_{B_{r, q}^{2 s, s}\left(\Omega^{T}\right)} \equiv\|u\|_{L_{r}\left(\Omega^{T}\right)}\right. & \\
+ & {\left[\int_{0}^{h_{0}}\left(\frac{\left\|\Delta_{x}(h, \Omega) D_{x}^{[2 s]} u\right\|_{L_{r}\left(\Omega^{T}\right)}}{h^{2 s-[2 s]}}\right)^{q} d h\right]^{1 / q} } \\
+ & {\left.\left[\int_{0}^{h_{0}}\left(\frac{\left\|\Delta_{t}(h,(0, T)) \partial_{t}^{[s]} u\right\|_{L_{r}\left(\Omega^{T}\right)}}{h^{s-[s]}}\right)^{q} d h\right]^{1 / q}<\infty\right\}, }
\end{aligned}
$$

where $s \in \mathbb{R}_{+} \cup\{0\}$ is noninteger, $1 \leq r, q \leq \infty$, and

$$
\begin{aligned}
B_{r, q}^{s}(\Omega)=\left\{u:\|u\|_{B_{r, q}^{s}(\Omega)} \equiv\right. & \|u\|_{L_{r}(\Omega)} \\
& \left.+\left[\int_{0}^{h_{0}}\left(\frac{\left\|\Delta_{x}(h, \Omega) D_{x}^{[s]} u\right\|_{L_{r}(\Omega)}}{h^{s-[s]}}\right)^{q} d h\right]^{1 / q}<\infty\right\},
\end{aligned}
$$

where

$$
\begin{array}{ll}
\Delta_{x}(h, \Omega) u(x, t)=u(x+h, t)-u(x, t), & x, x+h \in \Omega, \\
\Delta_{t}(h,(0, T)) u(x, t)=u(x, t+h)-u(x, t), & t, t+h \in(0, T) .
\end{array}
$$

Moreover, we define

$$
W_{r}^{2 s, s}\left(\Omega^{T}\right)=B_{r, r}^{2 s, s}\left(\Omega^{T}\right), \quad W_{r}^{s}(\Omega)=B_{r, r}^{s}(\Omega),
$$

which is possible in view of [8], where different kinds of differences were introduced.

Finally, we have

Lemma 2.2 (see [1]). Let $p>1, S_{1} \in C^{2 s}, f \in B_{r, q}^{2 s, s}\left(\Omega^{T}\right), g \in B_{r, q}^{2 s+1, s}\left(\Omega^{T}\right)$, $v(0) \in B_{r, q}^{2 s+2-2 / r}(\Omega), b_{i} \in B_{r, q}^{2 s+1-1 / r, s+1 / 2-1 /(2 r)}\left(S^{T}\right), i=1,2, b_{3} \in$ $B_{r, q}^{2 s+2-1 / r, s+1-1 /(2 r)}\left(S^{T}\right), s \in \mathbb{R}_{+}$noninteger, $1<q<\infty$. Suppose there exist functions $A, B \in B_{r, q}^{2 s, s}\left(\Omega^{T}\right)$, diam supp $A<\lambda$, where $\lambda$ is sufficiently small, such that the following compatibility conditions hold:

$$
D_{x}^{\alpha} \partial_{t}^{\beta+1} g-\operatorname{div}\left(D_{x}^{\alpha} \partial_{t}^{\beta} f\right)=\operatorname{div}\left(D_{x}^{\alpha} \partial_{t}^{\beta} B\right)+D_{x}^{\alpha} \partial_{t}^{\beta} A,
$$


for all $|\alpha|, \beta \geq 0$ such that $|\alpha|+2 \beta \leq[2 s]$. Then there exists a unique solution $(v, p)$ to problem (2.18) such that $v \in B_{r, q}^{2 s+2, s+1}\left(\Omega^{T}\right), \nabla p \in B_{r, q}^{2 s, s}\left(\Omega^{T}\right)$ and

$$
\begin{aligned}
& \|v\|_{B_{r, q}^{2 s+2, s+1}\left(\Omega^{T}\right)}+\|\nabla p\|_{B_{r, q}^{2 s, s}\left(\Omega^{T}\right)} \leq c\left(\|f\|_{B_{r, q}^{2 s, s}\left(\Omega^{T}\right)}\right. \\
& \quad+\|B\|_{B_{r, q}^{2 s, s}\left(\Omega^{T}\right)}+\|A\|_{B_{r, q}^{2 s, s}\left(\Omega^{T}\right)}+\|g\|_{B_{r, q}^{2 s+1, s}\left(\Omega^{T}\right)} \\
& \quad+\sum_{i=1}^{2}\left\|b_{i}\right\|_{B_{r, q}^{2 s+1-1 / r, s+1 / 2-1 /(2 r)}\left(S^{T}\right)}+\left\|b_{3}\right\|_{B_{r, q}^{2 s+2-1 / r, s+1-1 /(2 r)}\left(S^{T}\right)} \\
& \left.\quad+\|v(0)\|_{B_{r, q}^{2 s+2-2 / r}(\Omega)}+\sum_{i=0}^{[s-1]}\left\|\left.\partial_{t}^{i} f\right|_{t=0}\right\|_{B_{r, q}^{2 s-2 i-2 / r}(\Omega)}\right)
\end{aligned}
$$

3. Basic formulations. To prove Theorems 1 and 2 we formulate problems for quantities (1.38) which help us to improve the regularity of the weak solution.

Lemma 3.1. Let $^{(1)}=f_{, x_{3}}$. Then $\left(h^{(1)}, q^{(1)}\right)$ is a solution to the problem

$$
\begin{array}{ll}
h_{, t}^{(1)}-\operatorname{div} \mathbb{T}\left(h^{(1)}, q^{(1)}\right)=-v \cdot \nabla h^{(1)}-h^{(1)} \cdot \nabla v+g^{(1)} & \text { in } \Omega^{T}, \\
\operatorname{div} h^{(1)}=0 & \text { in } \Omega^{T}, \\
h^{(1)} \cdot \bar{n}=0, \quad \bar{n} \cdot \mathbb{D}\left(h^{(1)}\right) \cdot \bar{\tau}_{\alpha}=0, \quad \alpha=1,2, & \text { on } S_{1}^{T}, \\
h_{i}^{(1)}=0, \quad i=1,2, \quad h_{3, x_{3}}^{(1)}=0 & \text { on } S_{2}^{T}, \\
\left.h^{(1)}\right|_{t=0}=h^{(1)}(0) & \text { in } \Omega,
\end{array}
$$

where $\bar{n}$ is the normal vector to $S_{1}$ and $\bar{\tau}_{\alpha}, \alpha=1,2$, are tangent.

Proof. Equations $(3.1)_{1,2}$ and the boundary conditions $(3.1)_{3}$ follow directly from $(1.1)_{1,2}$ and $(1.1)_{3,4}$ by differentiating with respect to $x_{3}$ because $\Omega$ is a cylindrical domain with respect to the $x_{3}$ axis.

The boundary conditions (1.1) $)_{3,4}$ on $S_{2}$ assume the form

$$
v_{3}=0, \quad v_{i, x_{3}}+v_{3, x_{i}}=0, \quad i=1,2,
$$

so

$$
\left.v_{i, x_{3}}\right|_{S_{2}}=0, \quad i=1,2,
$$

which implies the first condition of $(3.1)_{4}$.

Expressing $(1.1)_{2}$ in the form

$$
v_{1, x_{1}}+v_{2, x_{2}}+v_{3, x_{3}}=0,
$$

we obtain, in view of (3.2),

$$
v_{3, x_{3} x_{3}}=-v_{1, x_{3} x_{1}}-v_{2, x_{3} x_{1}}=0 \quad \text { on } S_{2},
$$

which gives the second condition of $(3.1)_{4}$. This ends the proof. 
LEMMA 3.2. Let $q^{(1)}$ and $f_{3}$ be given. Then $w$ is a solution to the problem

$$
\begin{array}{ll}
w_{, t}+v \cdot \nabla w-\nu \Delta w=q^{(1)}+f_{3} & \text { in } \Omega^{T}, \\
w_{, n}=0 & \text { on } S_{1}^{T}, \\
w=0 & \text { on } S_{2}^{T}, \\
\left.w\right|_{t=0}=w(0) & \text { in } \Omega,
\end{array}
$$

where $\partial_{n}=\bar{n} \cdot \nabla$ and $\bar{n}$ is the normal vector to $S_{1}$.

Proof. Equation $(3.4)_{1}$ is exactly the third equation of $(1.1)_{1}$. Let

$$
\begin{aligned}
\left.\bar{n}\right|_{S_{1}} & =\frac{\nabla \varphi}{|\nabla \varphi|}=\frac{1}{|\nabla \varphi|}\left(\varphi_{, x_{1}}, \varphi_{, x_{2}}, 0\right), \\
\left.\bar{\tau}_{1}\right|_{S_{1}} & =\frac{\nabla^{\perp} \varphi}{|\nabla \varphi|}=\frac{1}{|\nabla \varphi|}\left(-\varphi, x_{2}, \varphi, x_{1}, 0\right),\left.\quad \bar{\tau}_{2}\right|_{S_{1}}=(0,0,1), \\
\left.\bar{n}\right|_{S_{2}} & =(0,0,1),\left.\quad \bar{\tau}_{1}\right|_{S_{2}}=(1,0,0),\left.\quad \bar{\tau}_{2}\right|_{S_{2}}=(0,1,0) .
\end{aligned}
$$

Condition $(3.4)_{3}$ follows from $(1.1)_{3}$. Expressing boundary conditions $(1.1)_{4}$ in the form

$$
\left.n_{i}\left(\frac{\partial v_{i}}{\partial x_{j}}+\frac{\partial v_{j}}{\partial x_{i}}\right) \tau_{\alpha j}\right|_{S_{1}}=0, \quad \alpha=1,2,
$$

where the summation convention over repeated indices is used, we have for $\alpha=2$ the equation

$$
\left.n_{i}\left(\frac{\partial v_{i}}{\partial x_{3}}+\frac{\partial v_{3}}{\partial x_{i}}\right)\right|_{S_{1}}=0 .
$$

Since $\left.\bar{n}\right|_{S_{1}}$ does not depend on $x_{3}$, the above condition implies $(3.4)_{2}$, and the proof is finished.

LEMmA 3.3. Let $F_{3}=(\operatorname{rot} f)_{3}, h^{(1)}, v$ and $w$ be given. Then $\chi=(\operatorname{rot} v)_{3}$ is a solution to the problem

$$
\begin{array}{ll}
\chi, t+v \cdot \nabla \chi-h_{3}^{(1)} \chi+h_{2}^{(1)} w_{, x_{1}}-h_{1}^{(1)} w_{, x_{2}}-\nu \Delta \chi=F_{3} & \text { in } \Omega^{T}, \\
\chi=v_{i}\left(n_{i, x_{j}} \tau_{1 j}+\tau_{1 i, x_{j}} n_{j}\right)+v \cdot \bar{\tau}_{1}\left(\tau_{12, x_{1}}-\tau_{11, x_{2}}\right) \equiv \chi_{*} & \text { on } S_{1}^{T}, \\
\chi_{, x_{3}}=0 & \text { on } S_{2}^{T}, \\
\left.\chi\right|_{t=0}=\chi(0) & \text { in } \Omega,
\end{array}
$$

where tangent and normal vectors to $S_{1}$ are defined by (3.5).

Proof. Differentiating $(1.1)_{2}$ with respect to $x_{1}$ and $(1.1)_{1}$ with respect to $x_{2}$, and subtracting the results we obtain $(3.8)_{1}$.

In view of (3.2) we have

$$
\chi_{, x_{3}}=v_{2, x_{1} x_{3}}-v_{1, x_{2} x_{3}}=0 \quad \text { on } S_{2},
$$

so $(3.8)_{3}$ holds. 
To show $(3.8)_{2}$, we express $v^{\prime}=\left(v_{1}, v_{2}\right)$ in a neighbourhood of $S_{1}$ in the form

$$
v^{\prime}=v \cdot \bar{\tau}_{1} \bar{\tau}_{1}+v \cdot \bar{n} \bar{n}
$$

where we have assumed that the vectors $\bar{\tau}_{1}, \bar{n}$ are defined in a neighbourhood of $S_{1}$. Continuing, we have

$$
\begin{aligned}
\left.\chi\right|_{S_{1}}= & \left(v \cdot \bar{\tau}_{1} \tau_{12}+v \cdot \bar{n} n_{2}\right)_{x_{1}}-\left.\left(v \cdot \bar{\tau}_{1} \tau_{11}+v \cdot \bar{n} n_{1}\right)_{, x_{2}}\right|_{S_{1}} \\
= & {\left[\left(v \cdot \bar{\tau}_{1}\right)_{x_{1}} \tau_{12}-\left(v \cdot \bar{\tau}_{1}\right)_{, x_{2}} \tau_{11}+(v \cdot \bar{n})_{x_{1}} n_{2}-(v \cdot \bar{n})_{x_{2}} n_{1}\right.} \\
& \left.+v \cdot \bar{\tau}_{1}\left(\tau_{12, x_{1}}-\tau_{11, x_{2}}\right)+v \cdot \bar{n}\left(n_{2, x_{1}}-n_{1, x_{2}}\right)\right]\left.\right|_{S_{1}} \\
= & {\left.\left[\bar{n} \cdot \nabla\left(v \cdot \bar{\tau}_{1}\right)-\bar{\tau}_{1} \cdot \nabla(v \cdot \bar{n})+v \cdot \bar{\tau}_{1}\left(\tau_{12, x_{1}}-\tau_{11, x_{2}}\right)\right]\right|_{S_{1}} } \\
= & {\left.\left[\bar{n} \cdot \nabla\left(v \cdot \bar{\tau}_{1}\right)+v \cdot \bar{\tau}_{1}\left(\tau_{12, x_{1}}-\tau_{11, x_{2}}\right)\right]\right|_{S_{1}} . }
\end{aligned}
$$

Writing (3.6) for $\alpha=1$ in the form

$$
\left.\left(-v_{i} n_{i, x_{j}} \tau_{1 j}+\bar{n} \cdot \nabla\left(v \cdot \bar{\tau}_{1}\right)-v_{j} \tau_{1 j, x_{i}} n_{i}\right)\right|_{S_{1}}=0
$$

and inserting it in (3.9) yields $(3.8)_{2}$. This concludes the proof.

Let $\Omega^{\prime}$ be a cross-section of $\Omega$ by the plane $P$ perpendicular to the $x_{3}$ axis. Then $\partial \Omega^{\prime}=S_{1} \cap P \equiv S_{1}^{\prime}$. Therefore, we can consider the problem

$$
\begin{array}{ll}
v_{2, x_{1}}-v_{1, x_{2}}=\chi & \text { in } \Omega^{\prime}, \\
v_{1, x_{1}}+v_{2, x_{2}}=-h_{3}^{(1)} & \text { in } \Omega^{\prime}, \\
v^{\prime} \cdot \bar{n}^{\prime}=0 & \text { on } S_{1},
\end{array}
$$

where $v^{\prime}=\left(v_{1}, v_{2}\right), \bar{n}^{\prime}=\frac{1}{|\nabla \varphi|}\left(\varphi_{, x_{1}}, \varphi_{, x_{2}}\right)$. In the case when $S_{1}^{\prime}$ is a circle, Lemma 3.3 takes the following form:

Lemma 3.4. Assume that $\Omega$ is a cylinder. Then $\chi$ satisfies $(3.8)_{1,4}$ and the boundary conditions

$$
\begin{aligned}
& \left.\chi\right|_{S_{1}}=\left.\frac{2 v \cdot \bar{e}_{\varphi}}{R}\right|_{S_{1}}, \\
& \left.\chi_{, x_{3}}\right|_{S_{2}}=0 .
\end{aligned}
$$

Proof. We have to prove $(3.11)_{1}$ only. To do this we consider $(3.8)_{2}$ on the circle $S_{1}^{\prime}$. Then $\bar{n}=\left(x_{1} / r, x_{2} / r\right), \bar{\tau}_{1}=\left(-x_{2} / r, x_{1} / r\right), r=\sqrt{x_{1}^{2}+x_{2}^{2}}$. Hence

$$
\begin{aligned}
& \tau_{12, x_{1}}-\tau_{11, x_{2}}=\frac{1}{r}, \quad v \cdot \bar{\tau}_{1}=-\frac{v_{1} x_{2}}{r}+\frac{v_{2} x_{1}}{r}, \quad n_{1, x_{j}} \tau_{1 j}=-\frac{x_{2}}{r^{2}}, \\
& \tau_{11, x_{j}} n_{j}=0, \quad n_{2, x_{j}} \tau_{1 j}=\frac{x_{1}}{r^{2}}, \quad \tau_{12, x_{j}} n_{j}=0 .
\end{aligned}
$$

Applying the expressions in $(3.8)_{2}$ yields $(3.11)_{1}$ and concludes the proof.

Finally, we formulate a problem for $h^{(2)}, q^{(2)}$. 
Lemma 3.5. Assume that $v, h^{(1)}, g^{(2)}=f_{x_{3} x_{3}}, v_{, x_{3} x_{3}}(0)$ are given. Then $\left(h^{(2)}, q^{(2)}\right)$ is a solution to the problem

$$
\begin{array}{ll}
h_{, t}^{(2)}-\operatorname{div} \mathbb{T}\left(h^{(2)}, q^{(2)}\right)=-h^{(2)} \cdot \nabla v-2 h^{(1)} \cdot \nabla h^{(1)} & \\
-v \cdot \nabla h^{(2)}+g^{(2)} & \text { in } \Omega^{T}, \\
\operatorname{div} h^{(2)}=0 & \text { in } \Omega^{T}, \\
\bar{n} \cdot h^{(2)}=0, \quad \bar{n} \cdot \mathbb{D}\left(h^{(2)}\right) \cdot \bar{\tau}_{\alpha}=0, \quad \alpha=1,2, & \text { on } S_{1}^{T}, \\
h_{3}^{(2)}=0, \quad h_{i, x_{3}}^{(2)}=f_{3, x_{i}}-g_{i}^{(1)} \equiv \bar{F}_{i}, \quad i=1,2, & \text { on } S_{2}^{T}, \\
\left.h^{(2)}\right|_{t=0}=h^{(2)}(0) & \text { in } \Omega,
\end{array}
$$

where $F=\operatorname{rot} f, \bar{F}=\left(F_{1},-F_{2}, F_{3}\right)$.

Proof. (3.12) $1,2,3$ follow directly by differentiating (1.1) $)_{1,2,3,4}$ with respect to $x_{3}$. Now we calculate the boundary conditions on $S_{2}$. From $(1.1)_{3}$ we have

$$
\left.v_{3}\right|_{S_{2}}=0
$$

and $(1.1)_{4}$ implies

$$
\left.\frac{\partial v_{i}}{\partial x_{3}}\right|_{S_{2}}=0, \quad i=1,2 .
$$

Then the continuity equation $(1.1)_{2}$ yields

$$
\left.h_{3}^{(2)}\right|_{S_{2}}=\left.\frac{\partial^{2} v_{3}}{\partial x_{3}^{2}}\right|_{S_{2}}=-\left.\sum_{i=1}^{2} \frac{\partial v_{i, x_{3}}}{\partial x_{i}}\right|_{S_{2}}=0 .
$$

Therefore, the first boundary condition for $h^{(2)}$ on $S_{2}$ takes the form

$$
\left.h_{3}^{(2)}\right|_{S_{2}}=0 \text {. }
$$

Expressing $(1.1)_{1}$ in the explicit form

$$
v_{t}-\nu \Delta^{\prime} v-\nu \partial_{x_{3}}^{2} v+\nabla p=-v \cdot \nabla v+f
$$

where $\Delta^{\prime}=\partial_{x_{1}}^{2}+\partial_{x_{2}}^{2}$, taking the first two components of (3.16), differentiating them with respect to $x_{3}$, projecting on $S_{2}$ and using (3.14) we obtain

$$
\left.h_{x_{3}}^{(2)^{\prime}}\right|_{S_{2}}=\left.\frac{1}{\nu} \nabla^{\prime} q^{(1)}\right|_{S_{2}}+\left.\frac{1}{\nu}\left(v \cdot \nabla h^{(1)^{\prime}}+h^{(1)} \cdot \nabla v^{\prime}-g^{(1)^{\prime}}\right)\right|_{S_{2}},
$$

where $\nabla^{\prime}=\left(\partial_{x_{1}}, \partial_{x_{2}}\right)$ and $u^{\prime}=\left(u_{1}, u_{2}\right)$. In view of (3.13) and (3.14) we have

$$
\left.\left(v \cdot \nabla h^{(1)^{\prime}}+h^{(1)} \cdot \nabla v^{\prime}\right)\right|_{S_{2}}=0 .
$$


Hence (3.17) takes the form

$$
\left.h_{, x_{3}}^{(2)^{\prime}}\right|_{S_{2}}=\left.\frac{1}{\nu}\left(\nabla^{\prime} q^{(1)}-g^{(1)^{\prime}}\right)\right|_{S_{2}} .
$$

Projecting the third component of (3.16) on $S_{2}$ and using (3.13), (3.14) we obtain

$$
\left.q^{(1)}\right|_{S_{2}}=-\left.v \cdot \nabla v \cdot \bar{n}\right|_{S_{2}}+\left.f_{3}\right|_{S_{2}}=\left.f_{3}\right|_{S_{2}} .
$$

Employing (3.19) in (3.18) yields

$$
\left.h_{, x_{3}}^{(2)^{\prime}}\right|_{S_{2}}=\left.\frac{1}{\nu}\left(\nabla^{\prime} f_{3}-g^{(1)^{\prime}}\right)\right|_{S_{2}} .
$$

This implies the second condition of $(3.12)_{4}$. This ends the proof.

4. Estimates. First we examine problem (3.8). Let us define a function $\tilde{\chi}$ to be a solution of the problem

$$
\begin{array}{ll}
\tilde{\chi}_{, t}-\nu \Delta \widetilde{\chi}=0 & \text { in } \Omega^{T}, \\
\tilde{\chi}=\chi_{*} & \text { on } S_{1}^{T}, \\
\tilde{\chi}, x_{3}=0 & \text { on } S_{2}^{T}, \\
\left.\tilde{\chi}\right|_{t=0}=0 & \text { in } \Omega .
\end{array}
$$

Then the function

$$
\chi^{\prime}=\chi-\tilde{\chi}
$$

is a solution to

$$
\begin{array}{ll}
\chi_{, t}^{\prime}+v \cdot \nabla \chi^{\prime}-h_{3}^{(1)} \chi^{\prime}+h_{2}^{(1)} w_{x_{1}}-h_{1}^{(1)} w_{x_{2}}-\nu \Delta \chi^{\prime} & \\
\quad=F_{3}-v \cdot \nabla \tilde{\chi}+h_{3}^{(1)} \tilde{\chi} & \text { in } \Omega^{T}, \\
\chi^{\prime}=0 & \text { on } S_{1}^{T}, \\
\chi_{, x_{3}}^{\prime}=0 & \text { on } S_{2}^{T}, \\
\left.\chi^{\prime}\right|_{t=0}=\chi(0) & \text { in } \Omega .
\end{array}
$$

First we replace $(1.17)_{2}$ by a more appropriate energy estimate

$$
\begin{aligned}
|v(t)|_{2, \Omega}+\left(\int_{0}^{t}\left\|v\left(t^{\prime}\right)\right\|_{W_{2}^{1, k}(\Omega)} d t^{\prime}\right)^{1 / 2} & \\
& \leq d_{2}(t)+\left|\partial_{x_{3}}^{k} v\right|_{2, \Omega^{t}} \equiv d_{5}(t), \quad t \leq T,
\end{aligned}
$$

where $k \in \mathbb{N}$ will be chosen later. Then (2.2) and (2.3) imply

$$
|v|_{\frac{2(4 k+1)}{2 k+1}, \Omega^{t}} \leq c d_{5}(t), \quad t \leq T \text {. }
$$


Lemma 4.1. Assume that $h_{3}^{(1)} \in L_{\infty, 1}\left(\Omega^{T}\right) \cap L_{\frac{10(4 k+1)}{18 k+7}}\left(\Omega^{T}\right), h^{(1)^{\prime}}=$ $\left(h_{1}^{(1)}, h_{2}^{(1)}\right) \in L_{\frac{2 k+1}{k}, \infty}\left(\Omega^{T}\right), F_{3} \in L_{\frac{2(4 k+1)}{6 k+1}}\left(\Omega^{T}\right), \tilde{\chi} \in W_{r}^{s, s / 2}\left(\Omega^{T}\right)$ with $\frac{5}{r}-$ $\frac{10 k}{4 k+1}+1 \leq s, k \geq 1, \chi(0) \in L_{2}(\Omega)$. Assume also that $v$ is a weak solution of (1.1) satisfying (4.4). Assume that $\left|\partial_{x_{3}}^{k} v\right|_{2, \Omega^{T}},\left|\partial_{x_{3}}^{k} \widetilde{\chi}\right|_{2, \Omega^{T}},\left|\nabla^{\prime} \partial_{x_{3}}^{k} v\right|_{2, \Omega^{T}}$ are finite. Then solutions of problem (3.8) satisfy

$$
\begin{aligned}
& |\chi|_{2, \infty, \Omega^{t}}+\|\chi\|_{L_{2}\left(0, t ; W_{2}^{1, k}(\Omega)\right)} \leq c \exp \left(c\left|h_{3}^{(1)}\right|_{\infty, 1, \Omega^{t}}\right) \\
& \quad \cdot\left[\left(d_{5}+\left|h_{3}^{(1)}\right|_{\frac{10(4 k+1)}{18 k+7}, \Omega^{t}}+1\right)\|\tilde{\chi}\|_{s, r, \Omega^{t}}+d_{2}\left|h^{(1)^{\prime}}\right|_{\frac{2 k+1}{k}, \infty, \Omega^{t}}\right. \\
& \left.\quad+\left|F_{3}\right|_{\frac{2(4 k+1)}{6 k+1}, \Omega^{t}}+|\chi(0)|_{2, \Omega}\right]+c\left|\partial_{x_{3}}^{k} \widetilde{\chi}\right|_{2, \Omega^{t}}+\left|\nabla^{\prime} \partial_{x_{3}}^{k} v\right|_{2, \Omega^{t}} \equiv A_{1}(t)
\end{aligned}
$$

for all $t \leq T$.

Proof. Multiplying (4.3) by $\chi^{\prime}$, integrating the result over $\Omega$ and using $(1.1)_{3}$ we get

$$
\begin{aligned}
\frac{1}{2} \frac{d}{d t}\left|\chi^{\prime}\right|_{2, \Omega}^{2}+\nu \mid \nabla & \left.\chi^{\prime}\right|_{2, \Omega} ^{2} \\
= & \int_{\Omega} h_{3}^{(1)} \chi^{\prime 2} d x-\int_{\Omega}\left(h_{2}^{(1)} w_{, x_{1}}-h_{1}^{(1)} w_{, x_{2}}\right) \chi^{\prime} d x \\
& \quad+\int_{\Omega} F_{3} \chi^{\prime} d x-\int_{\Omega} v \cdot \nabla \widetilde{\chi} \chi^{\prime} d x+\int_{\Omega} h_{3}^{(1)} \widetilde{\chi} \chi^{\prime} d x .
\end{aligned}
$$

Estimating the first term on the r.h.s. by $\left|h_{3}^{(1)}(t)\right|_{\infty, \Omega}\left|\chi^{\prime}\right|_{2, \Omega}^{2}$, applying the Poincaré inequality and integrating with respect to time yields

$$
\begin{aligned}
& \left|\chi^{\prime}(t)\right|_{2, \Omega}^{2}+\nu \int_{0}^{t}\left\|\chi^{\prime}\left(t^{\prime}\right)\right\|_{W_{2}^{1, k}(\Omega)}^{2} d t^{\prime} \leq c \exp \left(c\left|h_{3}^{(1)}\right|_{\infty, 1, \Omega^{t}}\right) \\
& \cdot\left[\int_{\Omega^{t}}\left|h^{(1)^{\prime}}\right|\left|w_{, x^{\prime}}\right|\left|\chi^{\prime}\right| d x d t^{\prime}+\left|\int_{\Omega^{t}} F_{3} \chi^{\prime} d x d t^{\prime}\right|+\left|\int_{\Omega^{t}} v \cdot \nabla \widetilde{\chi} \chi^{\prime} d x d t^{\prime}\right|\right. \\
& \left.\quad+\left|\int_{\Omega^{t}} h_{3}^{(1)} \tilde{\chi} \chi^{\prime} d x d t^{\prime}\right|+|\chi(0)|_{2, \Omega}^{2}\right]+c \int_{0}^{t}\left|\partial_{x_{3}}^{k} \chi^{\prime}\left(t^{\prime}\right)\right|_{2, \Omega}^{2} d t^{\prime},
\end{aligned}
$$

where $h^{(1)^{\prime}}=\left(h_{1}^{(1)}, h_{2}^{(1)}\right), w_{, x^{\prime}}=\left(w_{x_{1}}, w_{x_{2}}\right)$.

To estimate the first four integrals on the r.h.s. of (4.8) we use the imbedding (see (2.15))

$$
|u|_{\frac{2(4 k+1)}{2 k+1}, \Omega^{T}} \leq c\left(|u|_{2, \infty, \Omega^{T}}+\|u\|_{L_{2}\left(0, T ; W_{2}^{1, k}(\Omega)\right)}\right),
$$

valid for all $k \in \mathbb{N}$. 
By the Hölder inequality, the third integral on the r.h.s. of (4.8) is bounded by

$$
|v|_{\frac{2(4 k+1)}{2 k+1}, \Omega^{t}}|\nabla \widetilde{\chi}|_{\frac{4 k+1}{2 k}, \Omega^{t}}\left|\chi^{\prime}\right|_{\frac{2(4 k+1)}{2 k+1}, \Omega^{t}} \equiv I_{1}
$$

In view of (4.4) and (4.9), we have, for any $\varepsilon_{1}>0$,

$$
I_{1} \leq \varepsilon_{1}\left(\left|\chi^{\prime}(t)\right|_{2, \infty, \Omega^{t}}^{2}+\int_{0}^{t}\left\|\chi^{\prime}\left(t^{\prime}\right)\right\|_{W_{2}^{1, k}(\Omega)}^{2} d t^{\prime}\right)+c\left(1 / \varepsilon_{1}\right) d_{5}^{2}(t)|\nabla \widetilde{\chi}|_{\frac{\left.\right|_{k k+1} ^{2 k}, \Omega^{t}}{2}}^{2},
$$

where to estimate the last norm we apply the imbedding

$$
\|\nabla \widetilde{\chi}\|_{\frac{4 k+1}{2 k}\left(\Omega^{T}\right)} \leq c\|\widetilde{\chi}\|_{s, r, \Omega^{T}},
$$

which holds if

$$
\frac{5}{r}-\frac{10 k}{4 k+1}+1 \leq s, \quad s \in \mathbb{R}_{+}, r \in(1, \infty) .
$$

Using the imbedding (see [6, Ch. 3, Sec. 10])

$$
|u|_{2(2 k+1), \Omega} \leq c\|u\|_{W_{2}^{1, k}(\Omega)}, \quad \Omega \subset \mathbb{R}^{3},
$$

we estimate the first integral on the r.h.s. of (4.8) by

$$
\begin{aligned}
& \int_{0}^{t} d t^{\prime}\left[\varepsilon_{2}\left|\chi^{\prime}\left(t^{\prime}\right)\right|_{2(2 k+1), \Omega}^{2}+c\left(1 / \varepsilon_{2}\right)\left|h^{(1)^{\prime}}\right|_{\frac{2 k+1}{k}, \Omega}\left|w_{, x^{\prime}}\right|_{2, \Omega}^{2}\right] \\
& \leq \varepsilon_{2}\left(\left|\chi^{\prime}\right|_{2, \infty, \Omega^{t}}^{2}+\int_{0}^{t}\left\|\chi^{\prime}\left(t^{\prime}\right)\right\|_{W_{2}^{1, k}(\Omega)}^{2} d t^{\prime}\right)+c\left(1 / \varepsilon_{2}\right)\left|h^{(1)}\right|_{\frac{2 k+1}{k}, \infty, \Omega^{t}}^{2} d_{2}^{2}(t),
\end{aligned}
$$

for any $\varepsilon_{2}>0$, where $(1.17)_{2}$ was applied.

We bound the second term on the r.h.s. of (4.8) by

$$
\left|\chi^{\prime}\right|_{\frac{2(4 k+1)}{2 k+1}, \Omega^{t}}\left|F_{3}\right|_{\frac{2(4 k+1)}{6 k+1}, \Omega^{t}} \leq \varepsilon_{3}\left|\chi^{\prime}\right|_{\frac{2(4 k+1)}{2 k+1}, \Omega^{t}}^{2}+c\left(1 / \varepsilon_{3}\right)\left|F_{3}\right|_{\frac{2(4 k+1)}{6 k+1}, \Omega^{t}}^{2}
$$

for any $\varepsilon_{3}>0$. By the Hölder inequality, the fourth term on the r.h.s. of (4.8) is bounded by

$$
\begin{aligned}
\left|\chi^{\prime}\right|_{\frac{2(4 k+1)}{2 k+1}, \Omega^{t}}\left|h_{3}^{(1)} \widetilde{\chi}\right|_{\frac{2(4 k+1)}{6 k+1}, \Omega^{t}} & \\
& \leq\left|\chi^{\prime}\right|_{\frac{2(4 k+1)}{2 k+1}, \Omega^{t}}\left|h_{3}^{(1)}\right|_{\lambda_{1} \frac{2(4 k+1)}{6 k+1}, \Omega^{t}} \cdot|\widetilde{\chi}|_{\lambda_{2} \frac{2(4 k+1)}{6 k+1}, \Omega^{t}} \equiv I_{2},
\end{aligned}
$$

whenever $1 / \lambda_{1}+1 / \lambda_{2}=1$.

To estimate the last factor in $I_{2}$ by the r.h.s. of (4.10) with $r, s$ satisfying (4.11) we have to calculate $\lambda_{2}$ from the equality case of (4.11) and from the relation

$$
\frac{5}{r}-\frac{5}{\frac{2(4 k+1)}{6 k+1} \lambda_{2}}=s
$$


SO

$$
\frac{10 k}{4 k+1}-1=\frac{5(6 k+1)}{2(4 k+1) \lambda_{2}},
$$

which implies that $\lambda_{2}=\frac{5(6 k+1)}{2(6 k-1)}$ and $\lambda_{1}=\frac{5(6 k+1)}{18 k+7}$. Hence

$$
I_{2} \leq \varepsilon_{4}\left|\chi^{\prime}\right|_{\frac{2(4 k+1)}{2 k+1}, \Omega^{t}}^{2}+c\left(1 / \varepsilon_{4}\right)\left|h_{3}^{(1)}\right|_{\frac{10(4 k+1)}{18 k+7}, \Omega^{t}}^{2}\|\widetilde{\chi}\|_{s, r, \Omega^{t}}^{2},
$$

for any $\varepsilon_{4}>0$ and any $r, s$ satisfying (4.11).

Applying the above estimates in (4.8) and assuming that $\varepsilon_{1}$ to $\varepsilon_{4}$ are sufficiently small we obtain

$$
\begin{aligned}
\left|\chi^{\prime}(t)\right|_{2, \Omega}^{2}+ & \nu \int_{0}^{t}\left\|\chi^{\prime}\left(t^{\prime}\right)\right\|_{W_{2}^{1, k}(\Omega)}^{2} d t^{\prime} \leq c \exp \left(c\left|h_{3}^{(1)}\right|_{\infty, 1, \Omega^{t}}\right) \\
& \cdot\left[\left(d_{5}^{2}+\left|h_{3}^{(1)}\right|_{\frac{10(4 k+1)}{18 k+7}, \Omega^{t}}^{2}\right)\|\widetilde{\chi}\|_{s, r, \Omega^{t}}^{2}+d_{2}^{2}\left|h^{(1)^{\prime}}\right|_{\frac{2 k+1}{k}, \infty, \Omega^{t}}^{2}\right. \\
& \left.+\left|F_{3}\right|_{\frac{2(4 k+1)}{6 k+1}, \Omega^{t}}^{2}+|\chi(0)|_{2, \Omega}^{2}\right]+c \int_{0}^{t}\left|\partial_{x_{3}}^{k} \chi^{\prime}\left(t^{\prime}\right)\right|_{2, \Omega}^{2} d t^{\prime} \\
\equiv & A_{1}^{\prime}(t)+c \int_{0}^{t}\left|\partial_{x_{3}}^{k} \chi^{\prime}\left(t^{\prime}\right)\right|_{2, \Omega}^{2} d t^{\prime} .
\end{aligned}
$$

In view of transformation (4.2) we have

$$
\begin{aligned}
& |\chi(t)|_{2, \Omega}^{2}+\nu \int_{0}^{t}\left\|\chi\left(t^{\prime}\right)\right\|_{W_{2}^{1, k}(\Omega)}^{2} d t^{\prime} \leq A_{1}^{\prime}(t) \\
& \quad+c\left(|\widetilde{\chi}|_{2, \infty, \Omega^{t}}^{2}+\int_{0}^{t}\left\|\widetilde{\chi}\left(t^{\prime}\right)\right\|_{W_{2}^{1, k}(\Omega)}^{2} d t^{\prime}\right)+c \int_{0}^{t}\left|\partial_{x_{3}}^{k} \chi\left(t^{\prime}\right)\right|_{2, \Omega}^{2} d t^{\prime} \\
& \leq A_{1}^{\prime}(t)+c\left(|\widetilde{\chi}|_{2, \infty, \Omega^{t}}^{2}+\int_{0}^{t}\left\|\widetilde{\chi}\left(t^{\prime}\right)\right\|_{1, \Omega}^{2} d t^{\prime}\right)+c \int_{0}^{t}\left|\partial_{x_{3}}^{k} \widetilde{\chi}\left(t^{\prime}\right)\right|_{2, \Omega}^{2} d t^{\prime} \\
& \quad+c \int_{0}^{t}\left|\nabla^{\prime} \partial_{x_{3}}^{k} v\right|_{2, \Omega}^{2} d t^{\prime} .
\end{aligned}
$$

We need the imbedding

$$
|\widetilde{\chi}|_{2, \infty, \Omega^{T}}+\|\widetilde{\chi}\|_{L_{2}\left(0, T ; H^{1}(\Omega)\right)} \leq c\|\widetilde{\chi}\|_{s^{\prime}, r, \Omega^{T}},
$$

which holds for $r$ and $s^{\prime}$ such that

$$
\frac{5}{r}-\frac{3}{2}<s^{\prime}
$$


Since $\frac{10 k}{4 k+1}-1<\frac{3}{2}$, we see that $s^{\prime}$ can be chosen less than $s$. Therefore (4.14) implies (4.6), and this ends the proof.

From the assumption of Lemma 4.1 we have $\widetilde{\chi} \in W_{r}^{s, s / 2}\left(\Omega^{T}\right)$ whenever $s$ and $r$ satisfy (4.11). Then solving problem (4.1) we get

$$
\|\widetilde{\chi}\|_{s, r, \Omega^{T}} \leq c\left\|\chi_{*}\right\|_{s-1 / r, r, S_{1}^{T}}
$$

In view of $(3.8)_{1}$ or $(3.11)_{1}$ we see that $v$ must belong to $W_{r}^{s, s / 2}\left(\Omega^{T}\right)$ and

$$
\left\|\chi_{*}\right\|_{s-1 / r, r, S_{1}^{T}} \leq c\|v\|_{s, r, \Omega^{T}}
$$

Next we obtain an estimate for $h^{(1)}$. We recall that

$$
\int_{\Omega} h_{3}^{(1)} d x=\int_{\Omega} v_{3, x_{3}} d x=\int_{S_{2}\left(x_{3}=-a\right)} v_{3} d x-\int_{S_{2}\left(x_{3}=a\right)} v_{3} d x=0,
$$

and $h_{1}^{(1)}, h_{2}^{(1)}$ vanish on $S_{2}$. Hence for $h^{(1)}$ we have the Poincaré inequality

$$
|h|_{2, \Omega}^{(1)} \leq c\left|\nabla h^{(1)}\right|_{2, \Omega}
$$

LEMMA 4.2. Assume that $v$ is a weak solution to problem (1.1) satisfying (1.17). Assume that $h^{(1)} \in L_{\infty}\left(0, T ; L_{3}(\Omega)\right), g^{(1)} \in L_{2}\left(\Omega^{T}\right), f_{3} \in L_{2}\left(S_{2}^{T}\right)$, $h^{(1)}(0) \in L_{2}(\Omega)$. Then

$$
\begin{aligned}
\left|h^{(1)}(t)\right|_{2, \Omega}^{2}+\nu \int_{0}^{t}\left\|h^{(1)}\left(t^{\prime}\right)\right\|_{1, \Omega}^{2} d t^{\prime} \leq & c\left|h^{(1)}\right|_{3, \infty, \Omega^{t}}^{2} d_{2}^{2}(t)+c\left|f_{3}\right|_{2, S_{2}^{t}}^{2} \\
& +c\left|g^{(1)}\right|_{2, \Omega^{t}}^{2}+\left|h^{(1)}(0)\right|_{2, \Omega}^{2}
\end{aligned}
$$

for all $t \leq T$.

Proof. Multiplying $(3.1)_{1}$ by $h^{(1)}$ and integrating over $\Omega$ yields, for all $\varepsilon_{1}, \varepsilon_{2}, \varepsilon_{3}>0$,

$$
\begin{aligned}
\frac{1}{2} \frac{d}{d t} \mid & \left.h^{(1)}\right|_{2, \Omega} ^{2}+\nu\left\|h^{(1)}\right\|_{1, \Omega}^{2} \\
\leq & \int_{\Omega}\left|h^{(1)} \cdot \nabla v \cdot h^{(1)}\right| d x+\int_{\Omega}\left|g^{(1)} h^{(1)}\right| d x+\int_{S_{2}}\left|q^{(1)} h_{3}^{(1)}\right| d S_{2} \\
\leq & \varepsilon_{1}\left|h^{(1)}\right|_{6, \Omega}^{2}+c\left(1 / \varepsilon_{1}\right)\left|h^{(1)}\right|_{3, \Omega}^{2}|\nabla v|_{2, \Omega}^{2}+\varepsilon_{2}\left|h^{(1)}\right|_{2, \Omega}^{2} \\
& +c\left(1 / \varepsilon_{2}\right)\left|g^{(1)}\right|_{2, \Omega}^{2}+\varepsilon_{3}\left\|h^{(1)}\right\|_{1, \Omega}^{2}+c\left(1 / \varepsilon_{3}\right)\left|f_{3}\right|_{2, S_{2}}^{2},
\end{aligned}
$$

where (3.19) was used. Hence, after integration with respect to time, for sufficiently small $\varepsilon_{1}, \varepsilon_{2}$ and $\varepsilon_{3}$ we obtain the estimate (4.20). This ends the proof. 
The inequality (4.20) implies

$$
\begin{array}{r}
\left|h^{(1)}(t)\right|_{2, \Omega}^{2}+\nu \int_{0}^{t}\left\|h^{(1)}\left(t^{\prime}\right)\right\|_{W_{2}^{1, k}(\Omega)}^{2} d t^{\prime} \leq c\left|h^{(1)}\right|_{3, \infty, \Omega^{t}}^{2} d_{2}^{2}(t)+c\left|f_{3}\right|_{2, S_{2}^{t}}^{2} \\
+c\left|g^{(1)}\right|_{2, \Omega^{t}}^{2}+\left|h^{(1)}(0)\right|_{2, \Omega}^{2}+c\left|\partial_{x_{3}}^{k} h^{(1)}\right|_{2, \Omega^{t}}^{2} \equiv A_{2}(t), \quad t \leq T .
\end{array}
$$

Next we consider the problem

$$
\begin{array}{ll}
v_{1, x_{2}}-v_{2, x_{1}}=\chi & \text { in } \Omega^{\prime}, \\
v_{1, x_{1}}+v_{2, x_{2}}=-h_{3}^{(1)} & \text { in } \Omega^{\prime}, \\
v^{\prime} \cdot \bar{n}^{\prime}=0 & \text { on } S_{1}^{\prime},
\end{array}
$$

where $\Omega^{\prime}=\Omega \cap\left\{\right.$ plane $x_{3}=$ const $\left.\in(-a, a)\right\}, S_{1}^{\prime}=S_{1} \cap$ plane $x_{3}=$ const $\in(-a, a)\}$, and $x_{3}, t$ are treated as parameters.

Lemma 4.3. Let the assumptions of Lemmas 4.1 and 4.2 be satisfied. Let $\left|\partial_{x_{3}}^{k} h^{(1)}\right|_{2, \Omega^{T}}$ be finite, where $k \in \mathbb{N}$. Then the solutions of problem (4.23) satisfy

$$
\sup _{t^{\prime} \leq t}\left\|v^{\prime}\left(t^{\prime}\right)\right\|_{1, \Omega}+\left\|\nabla v^{\prime}\right\|_{L_{2}\left(0, t ; W_{2}^{1, k}(\Omega)\right)} \leq c\left(A_{1}(t)+A_{2}(t)\right)
$$

for all $t \leq T$, where $A_{1}$ is defined by (4.6) and $A_{2}$ by (4.22).

Proof. For solutions of problem (4.23) we get the estimates

$$
\begin{aligned}
& \left\|v^{\prime}\right\|_{1, \Omega^{\prime}}^{2} \leq c\left(|\chi|_{2, \Omega^{\prime}}^{2}+\left|h_{3}^{(1)}\right|_{2, \Omega^{\prime}}^{2}\right), \\
& \left\|v^{\prime}\right\|_{2, \Omega^{\prime}}^{2} \leq c\left(\|\chi\|_{1, \Omega^{\prime}}^{2}+\left\|h_{3}^{(1)}\right\|_{1, \Omega^{\prime}}^{2}\right),
\end{aligned}
$$

where $v^{\prime}=\left(v_{1}, v_{2}\right), \Omega^{\prime}$ is defined above,

$$
\|v\|_{k, \Omega^{\prime}}=\left(\sum_{\alpha_{1}+\alpha_{2} \leq k} \int_{\Omega^{\prime}}\left|\partial_{x_{1}}^{\alpha_{1}} \partial_{x_{2}}^{\alpha_{2}} v\right|^{2} d x_{1} d x_{2}\right)^{1 / 2}, \quad \alpha_{1}, \alpha_{2}, k \in \mathbb{N} \cup\{0\} .
$$

Integrating the above estimates with respect to $x_{3}$ and the second one also with respect to time, and adding them, we obtain

$$
\begin{aligned}
\sup _{t} \int_{-a}^{a}\left\|v^{\prime}\left(x_{3}, t^{\prime}\right)\right\|_{1, \Omega^{\prime}}^{2} d x_{3}+\int_{0}^{t} \int_{-a}^{a}\left\|v^{\prime}\left(x_{3}, t^{\prime}\right)\right\|_{2, \Omega^{\prime}}^{2} d x_{3} d t^{\prime} \\
\quad \leq c\left(\|\chi\|_{V_{2}^{0}\left(\Omega^{t}\right)}^{2}+\left\|h_{3}^{(1)}\right\|_{V_{2}^{0}\left(\Omega^{t}\right)}\right) .
\end{aligned}
$$

Adding (4.20) for $h^{(1)^{\prime}}=\left(h_{1}^{(1)}, h_{2}^{(1)}\right)$ and employing (4.20) to estimate the second term on the r.h.s. of (4.26), we obtain

$$
\begin{aligned}
& \sup _{t}\left\|v^{\prime}\right\|_{1, \Omega}^{2}+\int_{0}^{t}\left\|v^{\prime}\right\|_{2, \Omega}^{2} d t^{\prime} \leq c\|\chi\|_{V_{2}^{0}\left(\Omega^{t}\right)}^{2} \\
& \quad+c\left(d_{2}^{2}(t)\left|h^{(1)}\right|_{3, \infty, \Omega^{t}}+\left|g^{(1)}\right|_{2, \Omega^{t}}^{2}+\left|f_{3}\right|_{2, S_{2}^{t}}^{2}+\left|h^{(1)}(0)\right|_{2, \Omega}^{2}\right) .
\end{aligned}
$$


Applying estimates (4.6) and (4.22) to (4.27) gives (4.24). This concludes the proof.

Next we obtain an estimate for $v$ in terms of $h^{(1)}, h^{(2)}$.

Lemma 4.4. (i) Assume that $v$ is a weak solution to problem (1.1) such that the assumptions of Lemma 1.3 are satisfied.

(ii) Assume that $h^{(1)} \in L_{\infty, 1}\left(\Omega^{T}\right) \cap L_{54 / 17}\left(\Omega^{T}\right) \cap L_{3, \infty}\left(\Omega^{T}\right), h^{(i)} \in$ $L_{2}\left(0, T ; H^{1}(\Omega)\right), i=1,2$, and

$$
\begin{aligned}
\gamma_{0}(T)= & \left|h^{(1)}\right|_{\infty, 1, \Omega^{T}}+\left|h^{(1)}\right|_{54 / 17, \Omega^{T}}+\left|h^{(1)}\right|_{3, \infty, \Omega^{T}} \\
& +\sum_{i=1}^{2}\left\|h^{(i)}\right\|_{L_{2}\left(0, T ; H^{1}(\Omega)\right)}<\infty .
\end{aligned}
$$

(iii) Assume that $f \in L_{6 / 5, \infty}\left(\Omega^{T}\right) \cap L_{27 / 16}\left(\Omega^{T}\right), F_{3} \in L_{18 / 13}\left(\Omega^{T}\right), g^{(1)} \in$ $L_{2}\left(\Omega^{T}\right),\left.f_{3}\right|_{S_{2}} \in L_{2}\left(S_{2}^{T}\right)$,

$$
\begin{gathered}
G_{1}^{\prime}(T)=|f|_{6 / 5, \infty, \Omega^{T}}+|f|_{27 / 16, \Omega^{T}}+\left|F_{3}\right|_{18 / 13, \Omega^{T}} \\
+\left|g^{(1)}\right|_{2, \Omega^{T}}+\left|f_{3}\right|_{2, S_{2}^{T}}<\infty, \\
v(0) \in W_{27 / 16}^{22 / 27}(\Omega), \chi(0) \in L_{2}(\Omega), h^{(1)}(0) \in L_{2}(\Omega), \text { and } \\
G_{0}^{\prime}(0)=\|v(0)\|_{22 / 27,27 / 16, \Omega}+|\chi(0)|_{2, \Omega}+\left|h^{(1)}(0)\right|_{2, \Omega}<\infty .
\end{gathered}
$$

Then $v \in W_{27 / 16}^{2,1}\left(\Omega^{T}\right)$ and there exists an increasing positive function $\varphi_{0}=\varphi_{0}\left(\gamma_{0}(T), G_{1}^{\prime}(T), G_{0}^{\prime}(0)\right)($ see $(4.43))$ such that

$$
\|v\|_{2,27 / 16, \Omega^{T}} \leq \varphi_{0}\left(\gamma_{0}(T), G_{1}^{\prime}(T), G_{0}^{\prime}(0)\right) .
$$

Proof. In view of (2.1) and (2.2) we obtain from (4.24) the inequality

$$
\left|v^{\prime}\right|_{q, r, \Omega^{t}}+\left|v_{x}^{\prime}\right|_{q, r, \Omega^{t}} \leq c\left(A_{1}+A_{2}\right),
$$

which holds if

$$
\frac{2}{r}+\frac{2 k+1}{q k}=\frac{2 k+1}{2 k} .
$$

Hence

$$
\left\|v^{\prime}\right\|_{L_{r}\left(0, T ; W_{q}^{1}(\Omega)\right)} \leq c\left(A_{1}+A_{2}\right) .
$$

Let us use the imbedding

$$
\left|v^{\prime}\right|_{\sigma, \Omega} \leq c\left\|v^{\prime}\right\|_{1, q, \Omega},
$$

which holds if

$$
\frac{1}{q}=\frac{1}{3}+\frac{1}{\sigma} .
$$

Applying (4.32) in (4.31) yields

$$
\left|v^{\prime}\right|_{\sigma, r, \Omega^{T}} \leq c\left(A_{1}+A_{2}\right),
$$

provided 


$$
\frac{2}{r}+\left(2+\frac{1}{k}\right) \frac{1}{\sigma}=\frac{1}{3}+\frac{1}{6 k} .
$$

Inserting $\sigma=r$ in (4.34) we have

$$
r=\frac{6(4 k+1)}{2 k+1} .
$$

Hence (4.33) takes the form

$$
\left|v^{\prime}\right|_{\frac{6(4 k+1)}{2 k+1}, \Omega^{T}} \leq c\left(A_{1}+A_{2}\right) .
$$

To improve regularity of $v$ we consider the problem

$$
\begin{aligned}
& v_{, t}-\operatorname{div} \mathbb{T}(v, p)=-v^{\prime} \cdot \nabla v-w h^{(1)}+f, \\
& \operatorname{div} v=0, \\
& \left.v \cdot \bar{n}\right|_{S}=0,\left.\quad \bar{n} \cdot \mathbb{T}(v, p) \cdot \bar{\tau}_{\alpha}\right|_{S}=0, \quad \alpha=1,2, \\
& \left.v\right|_{t=0}=v(0) .
\end{aligned}
$$

Let us examine the regularity of the r.h.s. of $(4.36)_{1}$. By the Hölder inequality,

$$
\left|v^{\prime} \cdot \nabla v\right|_{\sigma, \Omega^{T}} \leq\left|v^{\prime}\right|_{\sigma \lambda_{1}, \Omega^{T}}|\nabla v|_{\sigma \lambda_{2}, \Omega^{T}} \equiv I_{1}
$$

with $1 / \lambda_{1}+1 / \lambda_{2}=1, \sigma \lambda_{1}=\frac{6(4 k+1)}{2 k+1}, \sigma \lambda_{2}=2$. Hence $\sigma=\frac{3(4 k+1)}{7 k+2}$ and

$$
I_{1} \leq c d_{2}\left(A_{1}+A_{2}\right) \text {. }
$$

Next

$$
\left|w h^{(1)}\right|_{\frac{3(4 k+1)}{7 k+2}, \Omega^{T}} \leq|w|_{\frac{3(4 k+1)}{7 k+2} \lambda_{1}^{\prime}, \Omega^{T}}\left|h^{(1)}\right|_{\frac{3(4 k+1)}{7 k+2} \lambda_{2}^{\prime}, \Omega^{T}} \equiv I_{2},
$$

whenever $1 / \lambda_{1}^{\prime}+1 / \lambda_{2}^{\prime}=1$.

Let $\frac{3(4 k+1)}{7 k+2} \lambda_{1}^{\prime}=\frac{2(4 k+1)}{2 k+1}$. Then $\lambda_{1}^{\prime}=\frac{2(7 k+2)}{3(2 k+1)}, \lambda_{2}^{\prime}=\frac{2(7 k+2)}{8 k+1}$ and

$$
I_{2} \leq c d_{5}\left|h^{(1)}\right|_{\frac{6(4 k+1)}{8 k+1}, \Omega^{T}},
$$

where (4.5) was employed.

Making use of the above considerations in (4.36) yields

$$
\|v\|_{2, \frac{3(4 k+1)}{7 k+2}, \Omega^{T}} \leq \Phi_{1}\|v\|_{s, r, \Omega^{T}}+\Phi_{2}
$$

whenever $s$ and $r$ satisfy (4.11) and

$$
\begin{aligned}
& \Phi_{1}=c d_{2}(T) \exp \left(c\left|h_{3}^{(1)}\right|_{\infty, 1, \Omega^{T}}\right)\left(d_{5}(T)+\left|h_{3}^{(1)}\right|_{\frac{10(4 k+1)}{18 k+7}, \Omega^{T}}+1\right), \\
& \Phi_{2}=c d_{2}(T) \exp \left(c\left|h_{3}^{(1)}\right|_{\infty, 1, \Omega^{T}}\right)\left(d_{2}(T)\left|h^{(1)^{\prime}}\right|_{\frac{2 k+1}{k}, \infty, \Omega^{T}}+\left|F_{3}\right|_{\frac{2(4 k+1)}{6 k+1}, \Omega^{T}}\right. \\
& \left.+|\chi(0)|_{2, \Omega}\right)+c\left(\left|\nabla^{\prime} \partial_{x_{3}}^{k} v\right|_{2, \Omega^{T}}+\left|h^{(1)}\right|_{3, \infty, \Omega^{T}}+\left|g^{(1)}\right|_{2, \Omega^{T}}\right. \\
& +\left|f_{3}\right|_{2, S_{2}^{T}}+\left|h^{(1)}(0)\right|_{2, \Omega}+\left|\partial_{x_{3}}^{k} h^{(1)}\right|_{2, \Omega^{T}}+d_{5}(T)\left|h^{(1)}\right|_{\frac{6(4 k+1)}{8 k+1}, \Omega^{T}}
\end{aligned}
$$

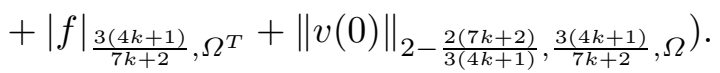


To obtain an estimate for $v$ from (4.37) we apply the interpolation inequality

$$
\|v\|_{W_{r}^{s, s / 2}\left(\Omega^{T}\right)} \leq \varepsilon^{1-\varkappa_{1}}\|v\|_{W_{\frac{3(4 k+1)}{7 k+2}}^{2,1}\left(\Omega^{T}\right)}+c \varepsilon^{-\varkappa_{1}}|v|_{2, \Omega^{T}},
$$

which holds for any $\varepsilon>0$ provided

$$
\varkappa_{1}=\left[\frac{5}{\frac{3(4 k+1)}{7 k+2}}-\left(\frac{5}{r}-s\right)\right] \frac{1}{2}<1,
$$

and $r, s$ satisfy (4.11), so $\frac{5}{r}-s=\frac{10 k}{4 k+1}-1=\frac{6 k-1}{4 k+1}$.

Now, the inequality in (4.40) holds if

$$
\frac{5 k+10}{3(4 k+1)}<1
$$

which is satisfied for $k>1$. Since $k \in \mathbb{N}$ we assume that $k=2$.

Hence $\varkappa_{1}=\frac{17 k+13}{6(4 k+1)}=\frac{47}{54}$. Therefore applying (4.39) in (4.37) yields

$$
\|v\|_{2,27 / 16, \Omega^{T}} \leq\left(\varepsilon^{7 / 54}\|v\|_{2,27 / 16, \Omega^{T}}+c \varepsilon^{-47 / 54} d_{2}\right) \Phi_{1}+\Phi_{2} .
$$

Taking $\varepsilon^{7 / 54} \Phi_{1}=\varepsilon_{1}$ implies that $\varepsilon=\left(\varepsilon_{1} / \Phi_{1}\right)^{54 / 7}$, so $\varepsilon^{-47 / 54}=\left(\Phi_{1} / \varepsilon_{1}\right)^{47 / 7}$. Finally, inserting $\varepsilon_{1}=1 / 2$ we obtain

$$
\|v\|_{2,27 / 16, \Omega^{T}} \leq c\left(\Phi_{1}^{54 / 7} d_{2}+\Phi_{2}\right)
$$

where

$$
\begin{aligned}
\Phi_{1}= & c d_{2}(T) \exp \left(c\left|h_{3}^{(1)}\right|_{\infty, 1, \Omega^{T}}\right)\left(d_{5}(T)+\left|h_{3}^{(1)}\right|_{90 / 43, \Omega^{T}}+1\right), \\
\Phi_{2}= & c d_{2}(T) \exp \left(c\left|h_{3}^{(1)}\right|_{\infty, 1, \Omega^{T}}\right)\left(d_{2}(T)\left|h^{(1)^{\prime}}\right|_{5 / 2, \infty, \Omega^{T}}\right. \\
& \left.+\left|F_{3}\right|_{18 / 13, \Omega^{T}}+|\chi(0)|_{2, \Omega}\right)+c\left(\left\|h^{(2)}\right\|_{L_{2}\left(0, T ; H^{1}(\Omega)\right)}\right. \\
& +\left|h_{3}^{(1)}\right|_{3, \infty, \Omega^{T}}+\left|g^{(1)}\right|_{2, \Omega^{T}}+\left|f_{3}\right|_{2, S_{2}^{T}} \\
& +\left|h^{(1)}(0)\right|_{2, \Omega}+\left|h^{(2)}\right|_{2, \Omega^{T}}+d_{5}(T)\left|h^{(1)}\right|_{54 / 17, \Omega^{T}} \\
& \left.+|f|_{27 / 16, \Omega^{T}}+\|v(0)\|_{22 / 27,27 / 16, \Omega}\right) .
\end{aligned}
$$

Making use of the form of $d_{2}$ and $d_{5}$ we obtain

$$
\begin{aligned}
\Phi_{1} \leq & c\left(|f|_{6 / 5, \infty, \Omega^{T}}+|v(0)|_{2, \Omega}\right) \exp \left(c\left|h^{(1)}\right|_{\infty, 1, \Omega^{T}}\right) \\
& \cdot\left(\left|h^{(1)}\right|_{54 / 17, \Omega^{T}}+\left\|h^{(1)}\right\|_{L_{2}\left(0, T ; H^{1}(\Omega)\right)}+|f|_{6 / 5, \infty, \Omega^{T}}+|v(0)|_{2, \Omega}+1\right) \\
\equiv & \Phi_{1}^{\prime}, \\
\Phi_{2} \leq & c\left(|f|_{6 / 5, \infty, \Omega^{T}}+|v(0)|_{2, \Omega}\right) \exp \left(c\left|h^{(1)}\right|_{\infty, 1, \Omega^{T}}\right) \\
& \cdot\left(\left|h^{(1)}\right|_{3, \infty, \Omega^{T}}^{2}+\left\|h^{(1)}\right\|_{L_{2}\left(0, T ; H^{1}(\Omega)\right)}^{2}+\left|F_{3}\right|_{18 / 13, \Omega^{T}}+|\chi(0)|_{2, \Omega}\right) \\
& +c\left(\left\|h^{(2)}\right\|_{L_{2}\left(0, T ; H^{1}(\Omega)\right)}+\left|h^{(1)}\right|_{3, \infty, \Omega^{T}}+\left\|h^{(1)}\right\|_{L_{2}\left(0, T ; H^{1}(\Omega)\right)}\right. \\
& +\left|h^{(1)}\right|_{54 / 17, \Omega^{T}}^{2}+|f|_{6 / 5, \infty, \Omega^{T}}^{2}+|v(0)|_{2, \Omega}^{2}
\end{aligned}
$$




$$
\begin{aligned}
& +\left|g^{(1)}\right|_{2, \Omega^{T}}+\left|f_{3}\right|_{2, S_{2}^{T}}+|f|_{27 / 16, \Omega^{T}} \\
& \left.+\left|h^{(1)}(0)\right|_{2, \Omega}+\|v(0)\|_{22 / 27,27 / 16, \Omega}\right) \equiv \Phi_{2}^{\prime} .
\end{aligned}
$$

Hence in view of assumptions (i) and (iii) we get from (4.42) the inequality

$$
\|v\|_{2,27 / 16, \Omega^{T}} \leq \Phi_{1}^{\prime} d_{2}+\Phi_{2}^{\prime} \leq \varphi_{0}\left(\gamma_{0}(T), G_{1}^{\prime}(T), G_{0}^{\prime}(0)\right),
$$

which concludes the proof.

In the assumption of Lemma 4.4 the quantity $h^{(2)} \in L_{2}\left(0, T ; H^{1}(\Omega)\right)$ appears. Therefore we need some estimates for $h^{(2)}$.

LEMma 4.5. Assume that $v$ is a weak solution of (1.1) satisfying (1.17). Assume that $h^{(2)}, h^{(1)} \in L_{3, \infty}\left(\Omega^{T}\right), f_{3} \in L_{2}\left(S_{2}^{T}\right), F^{\prime} \in L_{2}\left(S_{2}^{T}\right), g^{(1)} \in$ $L_{2}\left(S_{2}^{T}\right) \cap L_{2}\left(\Omega^{T}\right), h^{(2)}(0) \in L_{2}(\Omega), h^{(1)}(0) \in L_{2}(\Omega), g^{(2)} \in L_{2}\left(\Omega^{T}\right)$. Then solutions of (3.12) satisfy

$$
\begin{aligned}
& \left|h^{(2)}\right|_{2, \Omega}^{2}+\nu \int_{0}^{t}\left\|h^{(2)}\left(t^{\prime}\right)\right\|_{1, \Omega}^{2} d t^{\prime} \leq c d_{2}^{2}(t)\left|h^{(2)}\right|_{3, \infty, \Omega^{t}}^{2} \\
+ & c\left(\left|h^{(1)}\right|_{3, \infty, \Omega^{t}}^{2} d_{2}^{2}(t)+\left|g^{(1)}\right|_{2, \Omega^{t}}^{2}+\left|f_{3}\right|_{2, S_{2}^{t}}^{2}+\left|h^{(1)}(0)\right|_{2, \Omega}^{2}\right)\left|h^{(1)}\right|_{3, \infty, \Omega^{t}}^{2} \\
+ & c\left(\left|F^{\prime}\right|_{2, S_{2}^{t}}^{2}+\left|g^{(2)}\right|_{2, \Omega^{t}}^{2}\right)+\left|h^{(2)}(0)\right|_{2, \Omega}^{2}, \quad t \leq T .
\end{aligned}
$$

Proof. Multiplying $(3.12)_{1}$ by $h^{(2)}$ and integrating the result over $\Omega$ yields

$$
\begin{aligned}
& \frac{1}{2} \frac{d}{d t}\left|h^{(2)}\right|_{2, \Omega}^{2}+\nu\left|\mathbb{D}\left(h^{(2)}\right)\right|_{2, \Omega}^{2}-\int_{S} \bar{n} \cdot \mathbb{D}\left(h^{(2)}\right) \cdot \bar{\tau}_{\alpha} h^{(2)} \cdot \bar{\tau}_{\alpha} d S \\
& =-\int_{\Omega}\left(h^{(2)} \cdot \nabla v+2 h^{(1)} \cdot \nabla h^{(1)}+v \cdot \nabla h^{(2)}\right) h^{(2)} d x+\int_{\Omega} g^{(2)} h^{(2)} d x .
\end{aligned}
$$

Here the boundary term equals

$$
\int_{S_{2}}\left(\partial_{x_{3}} h_{\alpha}^{(2)}+\partial_{x_{\alpha}} h_{3}^{(2)}\right) h_{\alpha}^{(2)} d S_{2}=\int_{S_{2}} \partial_{x_{3}} h_{\alpha}^{(2)} h_{\alpha}^{(2)} d S_{2}=\int_{S_{2}} \bar{F}_{\alpha} h_{\alpha}^{(2)} d S \equiv I_{1},
$$

where in the first and second equality we used the first and second condition of $(3.12)_{4}$ respectively. Moreover, summation over $\alpha$ from 1 to 2 is understood. Finally, for all $\varepsilon_{1}>0$,

$$
\left|I_{1}\right| \leq \varepsilon_{1}\left\|h^{(2)}\right\|_{1, \Omega}^{2}+c\left(1 / \varepsilon_{1}\right)\left|F^{\prime}\right|_{2, S_{2}}^{2} .
$$

In view of the boundary conditions $\left.\bar{n} \cdot h^{(2)}\right|_{S_{1}}=0$, where we can choose for $\bar{n}$ two different linearly independent vectors (if we choose two different points on $S_{1}$ ), and $\left.h_{3}^{(2)}\right|_{S_{2}}=0$, we obtain the Korn inequality

$$
\left\|h^{(2)}\right\|_{1, \Omega} \leq c\left|\mathbb{D}\left(h^{(2)}\right)\right|_{2, \Omega} .
$$


In view of $(1.1)_{3}$, the first term on the r.h.s. of (4.45) equals

$$
I_{2}=-\int_{\Omega}\left(h^{(2)} \cdot \nabla v \cdot h^{(2)}+2 h^{(1)} \cdot \nabla h^{(1)} \cdot h^{(2)}\right) d x,
$$

so that, for all $\varepsilon_{2}>0$,

$$
\left|I_{2}\right| \leq \varepsilon_{2}\left|h^{(2)}\right|_{6, \Omega}^{2}+c\left(1 / \varepsilon_{2}\right)\left(|\nabla v|_{2, \Omega}^{2}\left|h^{(2)}\right|_{3, \Omega}^{2}+\left|\nabla h^{(1)}\right|_{2, \Omega}^{2}\left|h^{(1)}\right|_{3, \Omega}^{2}\right) .
$$

Making use of the above estimates in (4.45) and assuming that $\varepsilon_{1}, \varepsilon_{2}$ are sufficiently small we obtain

$$
\begin{aligned}
\frac{d}{d t}\left|h^{(2)}\right|_{2, \Omega}^{2}+\nu\left\|h^{(2)}\right\|_{1, \Omega}^{2} \leq & c\left(|\nabla v|_{2, \Omega}^{2}\left|h^{(2)}\right|_{3, \Omega}^{2}+\left|\nabla h^{(1)}\right|_{2, \Omega}^{2}\left|h^{(1)}\right|_{3, \Omega}^{2}\right) \\
& +c\left(\left|F^{\prime}\right|_{2, S_{2}}^{2}+\left|g^{(1)}\right|_{2, \Omega}^{2}+\left|g^{(2)}\right|_{2, \Omega}^{2}\right) .
\end{aligned}
$$

Integrating (4.47) with respect to time and using $(1.17)_{2}$ implies

$$
\begin{aligned}
\left|h^{(2)}\right|_{2, \Omega}^{2}+ & \nu \int_{0}^{t}\left\|h^{(2)}\left(t^{\prime}\right)\right\|_{1, \Omega}^{2} d t^{\prime} \\
\leq & c d_{2}^{2}(t)\left|h^{(2)}\right|_{3, \infty, \Omega^{t}}^{2}+c \int_{0}^{t}\left|\nabla h^{(1)}\left(t^{\prime}\right)\right|_{2, \Omega}^{2} d t^{\prime}\left|h^{(1)}\right|_{3, \infty, \Omega^{t}}^{2} \\
& +c\left(\left|F^{\prime}\right|_{2, S_{2}^{t}}^{2}+\left|g^{(1)}\right|_{2, \Omega^{t}}^{2}+\left|h^{(2)}(0)\right|_{2, \Omega}^{2}+\left|g^{(2)}\right|_{2, \Omega^{t}}^{2}\right) .
\end{aligned}
$$

Employing (4.20) in (4.48) yields (4.44), and this ends the proof.

In view of Lemmas 4.4 and 4.5 we obtain

$$
\|v\|_{2,27 / 16, \Omega^{T}} \leq \varphi_{1}\left(\gamma_{1}(T), G_{1}(T), G_{0}(0), T\right),
$$

where $\varphi_{1}$ is an increasing positive function and

$$
\begin{aligned}
& \gamma_{1}(T)=\left|h^{(1)}\right|_{\infty, 1, \Omega^{T}}+\left|h^{(1)}\right|_{3, \infty, \Omega^{T}} \\
&+\left|h^{(1)}\right|_{54 / 17, \Omega^{T}}+\left|h^{(2)}\right|_{3, \infty, \Omega^{T}}, \\
& G_{1}(T)=\sup _{t \leq T}|f(t)|_{6 / 5, \Omega}+|f|_{27 / 16, \Omega^{T}}+\left|F_{3}\right|_{18 / 13, \Omega^{T}} \\
&+\left|g^{(1)}\right|_{2, \Omega^{T}}+\left|F^{\prime}\right|_{2, S_{2}^{T}}+\left|f_{3}\right|_{2, S_{2}^{T}}+\left|g^{(2)}\right|_{2, \Omega^{T}}, \\
& G_{0}(0)=|v(0)|_{2, \Omega}+|\chi(0)|_{2, \Omega}+\left|h^{(1)}(0)\right|_{2, \Omega}+\left|h^{(2)}(0)\right|_{2, \Omega} \\
&+\|v(0)\|_{22 / 27,27 / 16, \Omega} .
\end{aligned}
$$

Let us introduce the space

$$
\mathfrak{M}_{0}\left(\Omega^{T}\right)=\left\{\left(h^{(1)}, h^{(2)}\right): \gamma_{1}(T)<\infty\right\} .
$$

Then (4.49) shows that the transformation

$$
\mathfrak{M}_{0}\left(\Omega^{T}\right) \ni\left(h^{(1)}, h^{(2)}\right) \mapsto v\left(h^{(1)}, h^{(2)}\right) \in W_{27 / 16}^{2,1}\left(\Omega^{T}\right),
$$


is well defined. Having this transformation, we consider problems (3.1) and (3.12), where $v$ is replaced by $v\left(h^{(1)}, h^{(2)}\right)$. Therefore, to prove the existence of solutions to problems (3.1) and (3.12) we need the following lemmas.

Lemma 4.6. Let $v \in W_{r}^{2,1}\left(\Omega^{T}\right), r>5 / 3$. Let $h^{(1)} \in L_{2}\left(\Omega^{T}\right), g^{(1)} \in$ $W_{\delta}^{\beta, \beta / 2}\left(\Omega^{T}\right), h^{(1)}(0) \in W_{\delta}^{2+\beta-2 / \delta}(\Omega)$, where $\beta>0, \delta>1$. Then solutions of (3.1) satisfy

$$
\begin{aligned}
& \left\|h^{(1)}\right\|_{2+\beta, \delta, \Omega^{T}}+\left\|\nabla q^{(1)}\right\|_{\beta, \delta, \Omega^{T}} \\
& \leq \varphi\left(\|v\|_{2, r, \Omega^{T}}\right)\left|h^{(1)}\right|_{2, \Omega^{T}}+c\left(\left\|g^{(1)}\right\|_{\beta, \delta, \Omega^{T}}+\left\|h^{(1)}(0)\right\|_{2+\beta-2 / \delta, \delta, \Omega}\right) .
\end{aligned}
$$

Proof. By Lemma 2.2 (see [1]) we have

$$
\begin{aligned}
& \left\|h^{(1)}\right\|_{2+\beta, \delta, \Omega^{T}}+\left\|\nabla q^{(1)}\right\|_{\beta, \delta, \Omega^{T}} \leq c\left(\left\|v \cdot \nabla h^{(1)}\right\|_{\beta, \delta, \Omega^{T}}\right. \\
& \left.+\left\|h^{(1)} \cdot \nabla v\right\|_{\beta, \delta, \Omega^{T}}+\left\|g^{(1)}\right\|_{\beta, \delta, \Omega^{T}}+\left\|h^{(1)}(0)\right\|_{2+\beta-2 / \delta, \delta, \Omega}\right) .
\end{aligned}
$$

Applying Lemma 2.2 from [19] to the first term on the r.h.s. of (4.53) yields

$$
\begin{aligned}
\left\|v \cdot \nabla h^{(1)}\right\|_{\beta, \delta, \Omega^{T}} \leq & \|v\|_{\beta+\varepsilon / \delta, \delta_{1}, \Omega^{T}}\left|\nabla h^{(1)}\right|_{\delta_{1}^{\prime}, \Omega^{T}} \\
& +\left\|\nabla h^{(1)}\right\|_{\beta+\varepsilon / \delta, \delta_{2}, \Omega^{T}}|v|_{\delta_{2}^{\prime}, \Omega^{T}} \equiv I_{1},
\end{aligned}
$$

whenever $\varepsilon>0$ and $1 / \delta_{i}+1 / \delta_{i}^{\prime}=1 / \delta, i=1,2$.

To estimate $I_{1}$ we use the imbeddings

$$
\|v\|_{\beta+\varepsilon / \delta, \delta_{1}, \Omega^{T}}+|v|_{\delta_{2}^{\prime}, \Omega^{T}} \leq c\|v\|_{2, r, \Omega^{T}},
$$

which hold for any $\varepsilon>0$ if

$$
\frac{5}{r}-\frac{5}{\delta_{1}}+\beta<2, \quad \frac{5}{r}-\frac{5}{\delta_{2}^{\prime}} \leq 2,
$$

and

$$
\begin{aligned}
& \left|\nabla h^{(1)}\right|_{\delta_{1}^{\prime}, \Omega^{T}} \leq \varepsilon_{1}^{1-\varkappa_{1}}\left\|h^{(1)}\right\|_{2+\beta, \delta, \Omega^{T}}+c \varepsilon_{1}^{-\varkappa_{1}^{\prime}}\left|h^{(1)}\right|_{2, \Omega^{T}}, \\
& \left\|\nabla h^{(1)}\right\|_{\beta+\varepsilon / \delta, \delta_{2}, \Omega^{T}} \leq \varepsilon_{2}^{1-\varkappa_{2}}\left\|h^{(1)}\right\|_{2+\beta, \delta, \Omega^{T}}+c \varepsilon_{2}^{-\varkappa_{2}^{\prime}}\left|h^{(1)}\right|_{2, \Omega^{T}},
\end{aligned}
$$

which hold for any $\varepsilon_{1}, \varepsilon_{2}>0$ if

$$
\begin{aligned}
& \varkappa_{1}=\left(\frac{5}{\delta}-\frac{5}{\delta_{1}^{\prime}}+1\right) \frac{1}{2+\beta}<1, \quad \varkappa_{1}^{\prime}=\left(\frac{5}{2}-\frac{5}{\delta_{1}^{\prime}}+1\right) \frac{1}{2+\beta}, \\
& \varkappa_{2}=\left(\frac{5}{\delta}-\frac{5}{\delta_{2}}+\beta+\frac{\varepsilon}{\delta}+1\right) \frac{1}{2+\beta}<1, \\
& \varkappa_{2}^{\prime}=\left(\frac{5}{2}-\frac{5}{\delta_{2}}+\beta+\frac{\varepsilon}{\delta}+1\right) \frac{1}{2+\beta} .
\end{aligned}
$$

The inequalities (4.56) and (4.58) imply the restrictions

$$
\frac{5}{r}+\beta<2+\frac{5}{\delta_{1}}<3+\beta, \quad \frac{5}{r} \leq 2+\frac{5}{\delta_{2}^{\prime}}<3 .
$$


By Lemma 2.2 from [19], the second term on the r.h.s. of (4.53) is estimated in the following way:

$$
\begin{aligned}
\left\|h^{(1)} \cdot \nabla v\right\|_{\beta, \delta, \Omega^{T}} \leq & \|\nabla v\|_{\beta+\varepsilon / \delta, \delta_{3}, \Omega^{T}}\left|h^{(1)}\right|_{\delta_{3}^{\prime}, \Omega^{T}} \\
& +\left\|h^{(1)}\right\|_{\beta+\varepsilon / \delta, \delta_{4}, \Omega^{T}}|\nabla v|_{\delta_{4}^{\prime}, \Omega^{T}} \equiv I_{2}
\end{aligned}
$$

whenever $\varepsilon>0$ and $1 / \delta_{i}+1 / \delta_{i}^{\prime}=1 / \delta, i=3,4$.

To estimate $I_{2}$ we make use of the imbeddings

$$
\|\nabla v\|_{\beta+\varepsilon / \delta, \delta_{3}, \Omega^{T}}+|\nabla v|_{\delta_{4}^{\prime}, \Omega^{T}} \leq c\|v\|_{2, r, \Omega^{T}},
$$

which hold if

$$
\frac{5}{r}-\frac{5}{\delta_{3}}+\beta+1<2, \quad \frac{5}{r}-\frac{5}{\delta_{4}^{\prime}}+1 \leq 2,
$$

and the interpolation inequalities

$$
\begin{aligned}
& \left|h^{(1)}\right|_{\delta_{3}^{\prime}, \Omega^{T}} \leq \varepsilon_{3}^{1-\varkappa_{3}}\left\|h^{(1)}\right\|_{2+\beta, \delta, \Omega^{T}}+c \varepsilon_{3}^{-\varkappa_{3}^{\prime}}\left|h^{(1)}\right|_{2, \Omega^{T}}, \\
& \left\|h^{(1)}\right\|_{\beta+\varepsilon / \delta, \delta_{4}, \Omega^{T}} \leq \varepsilon_{4}^{1-\varkappa_{4}}\left\|h^{(1)}\right\|_{2+\beta, \delta, \Omega^{T}}+c \varepsilon_{4}^{-\varkappa_{4}^{\prime}}\left|h^{(1)}\right|_{2, \Omega^{T}},
\end{aligned}
$$

which hold for any $\varepsilon_{3}>0$ if

$$
\begin{aligned}
& \varkappa_{3}=\left(\frac{5}{\delta}-\frac{5}{\delta_{3}^{\prime}}\right) \frac{1}{2+\beta}<1, \quad \varkappa_{3}^{\prime}=\left(\frac{5}{2}-\frac{5}{\delta_{3}^{\prime}}\right) \frac{1}{2+\beta}, \\
& \varkappa_{4}=\left(\frac{5}{\delta}-\frac{5}{\delta_{4}}+\beta+\frac{\varepsilon}{\delta}\right) \frac{1}{2+\beta}<1, \\
& \varkappa_{4}^{\prime}=\left(\frac{5}{2}-\frac{5}{\delta_{4}}+\beta+\frac{\varepsilon}{\delta}\right) \frac{1}{2+\beta} .
\end{aligned}
$$

From (4.62) and (4.64) we obtain the restrictions

$$
\frac{5}{r}+\beta<1+\frac{5}{\delta_{3}}<3+\beta, \quad \frac{5}{r}<1+\frac{5}{\delta_{4}^{\prime}}<3 .
$$

From (4.59) and (4.65) we have $r>5 / 3$. Employing the above estimates in (4.53) and assuming that $\varepsilon_{i}, i=1, \ldots, 4$, are sufficiently small we obtain (4.52). This ends the proof.

LEMma 4.7. Let $v \in W_{r}^{2,1}\left(\Omega^{T}\right), r>5 / 3, h^{(1)}, h^{(2)} \in L_{2}\left(\Omega^{T}\right), h^{(1)} \in$ $W_{\delta}^{2+\beta, 1+\beta / 2}\left(\Omega^{T}\right), g^{(2)} \in W_{\delta}^{\beta, \beta / 2}\left(\Omega^{T}\right), F^{\prime} \in W_{\delta}^{1+\beta-1 / \delta, 1 / 2+1 / 2 \beta-1 /(2 \delta)}\left(S_{2}^{T}\right)$, $h^{(2)}(0) \in W_{\delta}^{2+\beta-2 / \delta}(\Omega)$. Assume that $\beta>0$ and $\delta>1$ satisfy $5 / \delta<3+\beta$. Then solutions of problem (3.12) satisfy

$$
\begin{aligned}
\| h^{(2)} & \left\|_{2+\beta, \delta, \Omega^{T}}+\right\| \nabla q^{(2)} \|_{\beta, \delta, \Omega^{T}} \\
\leq & \varphi\left(\|v\|_{2, r, \Omega^{T}}\right)\left|h^{(2)}\right|_{2, \Omega^{T}}+\varphi\left(\left\|h^{(1)}\right\|_{2+\beta, \delta, \Omega^{T}}\right)\left|h^{(1)}\right|_{2, \Omega^{T}} \\
& +c\left(\left\|g^{(2)}\right\|_{\beta, \delta, \Omega^{T}}+\left\|F^{\prime}\right\|_{1+\beta-1 / \delta, \delta, S_{2}^{T}}+\left\|h^{(2)}(0)\right\|_{2+\beta-2 / \delta, \delta, \Omega}\right) .
\end{aligned}
$$


Proof. Lemma 2.2 (see [1]) yields

$$
\begin{aligned}
\left\|h^{(2)}\right\|_{2+\beta, \delta, \Omega^{T}}+\left\|\nabla q^{(2)}\right\|_{\beta, \delta, \Omega^{T}} & \\
\leq & c\left(\left\|v \cdot \nabla h^{(2)}\right\|_{\beta, \delta, \Omega^{T}}+\left\|h^{(1)} \cdot \nabla h^{(1)}\right\|_{\beta, \delta, \Omega^{T}}+\left\|h^{(2)} \cdot \nabla v\right\|_{\beta, \delta, \Omega^{T}}\right. \\
& \left.+\left\|g^{(2)}\right\|_{\beta, \delta, \Omega^{T}}+\left\|h^{(2)}(0)\right\|_{2+\beta-2 / \delta, \delta, \Omega}+\left\|F^{\prime}\right\|_{1+\beta-1 / \delta, \delta, S_{2}^{T}}\right) .
\end{aligned}
$$

Applying Lemma 2.2 from [19] to the first and third terms on the r.h.s. of (4.67) yields (4.54)-(4.65) with $h^{(1)}$ replaced by $h^{(2)}$.

We examine the second term on the r.h.s. of (4.67) in the following way:

$$
\begin{aligned}
\left\|h^{(1)} \cdot \nabla h^{(1)}\right\|_{\beta, \delta, \Omega^{T}} \leq & \left\|h^{(1)}\right\|_{\beta+\varepsilon / \delta, \delta_{5}, \Omega^{T}}\left|\nabla h^{(1)}\right|_{\delta_{5}^{\prime}, \Omega^{T}} \\
& +\left\|\nabla h^{(1)}\right\|_{\beta+\varepsilon / \delta, \delta_{6}, \Omega^{T}}\left|h^{(1)}\right|_{\delta_{6}^{\prime}, \Omega^{T}} \equiv I_{3}
\end{aligned}
$$

whenever $1 / \delta_{i}+1 / \delta_{i}^{\prime}=1 / \delta, i=5,6$, and $\varepsilon>0$.

The imbedding

$$
\left\|h^{(1)}\right\|_{\beta+\varepsilon / \delta, \delta_{5}, \Omega^{T}}+\left\|\nabla h^{(1)}\right\|_{\beta+\varepsilon / \delta, \delta_{6}, \Omega^{T}} \leq c\left\|h^{(1)}\right\|_{2+\beta, \delta, \Omega^{T}}
$$

and the interpolation inequality

$$
\left|h^{(1)}\right|_{\delta_{6}^{\prime}, \Omega^{T}}+\left|\nabla h^{(1)}\right|_{\delta_{5}^{\prime}, \Omega^{T}} \leq \bar{\varepsilon}_{1}\left\|h^{(1)}\right\|_{2+\beta, \delta, \Omega^{T}}+c\left(1 / \bar{\varepsilon}_{1}\right)\left|h^{(1)}\right|_{2, \Omega^{T}},
$$

which both hold for

$$
5 / \delta<3+\beta,
$$

imply that

$$
I_{3} \leq \varepsilon_{5}\left\|h^{(1)}\right\|_{2+\beta, \delta, \Omega^{T}}+\varphi\left(1 / \varepsilon_{5},\left\|h^{(1)}\right\|_{2+\beta, \delta, \Omega^{T}}\right)\left|h^{(1)}\right|_{2, \Omega^{T}} .
$$

In view of the above considerations, inequality (4.66) follows for sufficiently small $\varepsilon_{1}, \ldots, \varepsilon_{5}$ (see Lemma 4.6). This ends the proof.

Repeating the proof of Lemma 3.4 from [19] we obtain

$$
\begin{aligned}
\left|h^{(1)}\right|_{2, \Omega^{t}} \leq & c\left[|\nabla v|_{3,2, \Omega^{t}} \exp \left(c|\nabla v|_{3,2, \Omega^{t}}^{2}\right)+1\right] \\
& \cdot\left[\left|g^{(1)}\right|_{2, \Omega^{t}}+\left|f_{3}\right|_{2, S_{2}}^{2}+\left|h^{(1)}(0)\right|_{2, \Omega}\right], \quad t \leq T,
\end{aligned}
$$

and

$$
\begin{aligned}
\left|h^{(1)}(t)\right|_{2, \Omega} \leq & \exp \left(c|\nabla v|_{3,2, \Omega^{t}}^{2}\right) \\
& \cdot\left[\left|g^{(1)}\right|_{2, \Omega^{t}}+\left|f_{3}\right|_{2, S_{2}^{t}}^{2}+e^{-\nu t}\left|h^{(1)}(0)\right|_{2, \Omega}\right], \quad t \leq T .
\end{aligned}
$$

Finally, we shall obtain energy type estimates for solutions of problem (3.12). For this purpose we express (4.47) in the form

$$
\begin{aligned}
\frac{d}{d t}\left|h^{(2)}\right|_{2, \Omega}^{2}+\nu\left\|h^{(2)}\right\|_{1, \Omega}^{2} & \leq c\left(|\nabla v|_{3, \Omega}^{2}\left|h^{(2)}\right|_{2, \Omega}^{2}\right. \\
+ & \left.\left|\nabla h^{(1)}\right|_{3, \Omega}^{2}\left|h^{(1)}\right|_{2, \Omega}^{2}\right)+\left(\left|F^{\prime}\right|_{2, S_{2}}^{2}+\left|g^{(2)}\right|_{2, \Omega}^{2}\right) .
\end{aligned}
$$

This implies 
(4.73)

$$
\begin{aligned}
\frac{d}{d t}\left(\left|h^{(2)}\right|_{2, \Omega}^{2} e^{\left.\nu t-c|\nabla v|_{3,2, \Omega^{t}}^{2}\right) \leq}\right. & c\left[\left|\nabla h^{(1)}\right|_{3, \Omega}^{2}\left|h^{(1)}\right|_{2, \Omega}^{2}\right. \\
& +\left(\left|F^{\prime}\right|_{2, S_{2}}^{2}+\left|g^{(2)}\right|_{2, \Omega}^{2}\right] e^{\nu t-c|\nabla v|_{3,2, \Omega}^{2}} .
\end{aligned}
$$

Integrating (4.73) with respect to time yields

$$
\begin{aligned}
\left|h^{(2)}(t)\right|_{2, \Omega}^{2} \leq & \exp \left(c|\nabla v|_{3,2, \Omega^{t}}^{2}\right)\left[c \left(\sup _{t}\left|h^{(1)}(t)\right|_{2, \Omega}^{2}\left|\nabla h^{(1)}\right|_{3,2, \Omega^{t}}^{2}\right.\right. \\
& \left.\left.+\left|F^{\prime}\right|_{2, S_{2}^{t}}^{2}+\left|g^{(2)}\right|_{2, \Omega^{t}}^{2}\right)+e^{-\nu t}\left|h^{(2)}(0)\right|_{2, \Omega}^{2}\right] .
\end{aligned}
$$

Making use of (4.71) in (4.74) gives

$$
\begin{aligned}
\left|h^{(2)}(t)\right|_{2, \Omega}^{2} \leq & \exp \left(c|\nabla v|_{3,2, \Omega^{t}}^{2}\right) \\
& \cdot\left[c\left(\left|\nabla h^{(1)}\right|_{3,2, \Omega^{t}}^{2}\left|g^{(1)}\right|_{2, \Omega^{t}}+\left|f_{3}\right|_{2, S_{2}^{t}}^{2}+\left|h^{(1)}(0)\right|_{2, \Omega}^{2}\right)\right. \\
& \left.\left.+\left|F^{\prime}\right|_{2, S_{2}^{t}}^{2}+\left|g^{(2)}\right|_{2, \Omega^{t}}^{2}\right)+e^{-\nu t}\left|h^{(2)}(0)\right|_{2, \Omega}^{2}\right] .
\end{aligned}
$$

Integrating (4.72) with respect to time and using (4.71) and (4.75) yields (4.76) $\left|h^{(2)}\right|_{2, \Omega^{t}}^{2} \leq c \exp \left(c|\nabla v|_{3,2, \Omega^{t}}^{2}\right)$

$$
\begin{aligned}
& \cdot\left[\left|\nabla h^{(1)}\right|_{3,2, \Omega^{t}}^{2}\left(\left|g^{(1)}\right|_{2, \Omega^{t}}^{2}+\left|f_{3}\right|_{2, S_{2}^{t}}^{2}+\left|h^{(1)}(0)\right|_{2, \Omega}^{2}\right)\right. \\
& \left.+\left|F^{\prime}\right|_{2, S_{2}^{t}}^{2}+\left|g^{(2)}\right|_{2, \Omega^{t}}+\left|h^{(2)}(0)\right|_{2, \Omega}^{2}\right]\left(1+|\nabla v|_{3,2, \Omega^{t}}^{2}\right) .
\end{aligned}
$$

5. Local existence and uniqueness. To prove the existence of solutions to problem (1.1), we apply transformation (4.51) to examine the following problems:

$$
\begin{aligned}
& h_{t}^{(1)}-\operatorname{div} \mathbb{T}\left(h^{(1)}, q^{(1)}\right)=-\lambda\left[v\left(\widetilde{h}^{(1)}, \widetilde{h}^{(2)}, \bar{v}\right) \cdot \nabla \widetilde{h}^{(1)}\right. \\
& \left.+\widetilde{h}^{(1)} \cdot \nabla v\left(\widetilde{h}^{(1)}, \widetilde{h}^{(2)}, \bar{v}\right)\right]+g^{(1)} \quad \text { in } \Omega^{T}, \\
& \operatorname{div} h^{(1)}=0 \quad \text { in } \Omega^{T}, \\
& h^{(1)} \cdot \bar{n}=0, \quad \bar{n} \cdot \mathbb{D}\left(h^{(1)}\right) \cdot \bar{\tau}_{\alpha}=0, \quad \alpha=1,2, \quad \text { on } S_{1}^{T}, \\
& h_{i}^{(1)}=0, \quad i=1,2, \quad h_{3, x_{3}}^{(1)}=0 \quad \text { on } S_{2}^{T} \text {, } \\
& \left.h^{(1)}\right|_{t=0}=h^{(1)}(0) \quad \text { in } \Omega \text {, }
\end{aligned}
$$

and

$$
\begin{aligned}
& h_{, t}^{(2)}-\operatorname{div} \mathbb{T}\left(h^{(2)}, q^{(2)}\right)=-\lambda\left[v\left(\widetilde{h}^{(1)}, \widetilde{h}^{(2)}, \bar{v}\right) \cdot \nabla \widetilde{h}^{(2)}\right. \\
& \left.+\widetilde{h}^{(2)} \cdot \nabla v\left(\widetilde{h}^{(1)}, \widetilde{h}^{(2)}, \bar{v}\right)\right]+g^{(2)} \quad \text { in } \Omega^{T}, \\
& \operatorname{div} h^{(2)}=0 \\
& \bar{n} \cdot h^{(2)}=0, \quad \bar{n} \cdot \mathbb{D}\left(h^{(2)}\right) \cdot \bar{\tau}_{\alpha}=0, \quad \alpha=1,2, \quad \text { on } S_{1}^{T}, \\
& h_{, x_{3}}^{(2)^{\prime}}=\bar{F}^{\prime}, \quad h_{3}^{(2)}=0 \\
& \left.h^{(2)}\right|_{t=0}=h^{(2)}(0) \\
& \text { on } S_{2}^{T} \text {, } \\
& \text { in } \Omega \text {, }
\end{aligned}
$$


where $\lambda \in[0,1]$ and $\bar{v}$ is the weak solution from Lemma 1.4. Problems (5.1) and (5.2) lead to the mapping

$$
\left(h^{(1)}, h^{(2)}\right)=\Phi\left(\widetilde{h}^{(1)}, \widetilde{h}^{(2)}, \bar{v}, \lambda\right) .
$$

The main problem of this section is to show the existence of a fixed point of transformation (5.3) for $\lambda=1$, together with an estimate. The above presentation suggests using the Leray-Schauder fixed point theorem.

To define a domain of mapping (5.3) we take into account (4.51) and also the proofs of Lemmas 4.6 and 4.7. In view of the interpolation inequalities (4.57) and (4.63) for $h^{(1)}$ and $h^{(2)}$, we define the space

$$
\mathfrak{M}\left(\Omega^{T}\right)=\mathfrak{M}_{0}\left(\Omega^{T}\right) \cap \mathfrak{M}_{1}\left(\Omega^{T}\right),
$$

where

$$
\begin{aligned}
\mathfrak{M}_{1}\left(\Omega^{T}\right)=\left\{\left(h^{(1)}, h^{(2)}\right):\right. & h^{(1)}, h^{(2)} \in L_{\delta_{3}^{\prime}}\left(\Omega^{T}\right) \cap W_{\delta_{4}}^{\beta+\varepsilon / \delta, \beta / 2+\varepsilon /(2 \delta)}\left(\Omega^{T}\right), \\
& h^{(1)} \in L_{\delta_{6}^{\prime}}\left(\Omega^{T}\right) \cap W_{\delta_{5}}^{\beta+\varepsilon / \delta, \beta / 2+\varepsilon /(2 \delta)}\left(\Omega^{T}\right), \\
& \nabla h^{(1)}, \nabla h^{(2)} \in L_{\delta_{1}^{\prime}}\left(\Omega^{T}\right) \cap W_{\delta_{2}}^{\beta+\varepsilon / \delta, \beta / 2+\varepsilon /(2 \delta)}\left(\Omega^{T}\right), \\
& \left.\nabla h^{(1)} \in L_{\delta_{5}^{\prime}}\left(\Omega^{T}\right) \cap W_{\delta_{6}}^{\beta+\varepsilon / \delta, \beta / 2+\varepsilon /(2 \delta)}\left(\Omega^{T}\right)\right\}
\end{aligned}
$$

with

$$
\begin{aligned}
& \frac{5}{r}+\beta+\frac{5}{\delta_{3}^{\prime}}<1+\frac{5}{\delta}<3+\beta+\frac{5}{\delta_{3}^{\prime}} \\
& \frac{5}{r}+\frac{5}{\delta_{4}}<1+\frac{5}{\delta}<3+\frac{5}{\delta_{4}}, \\
& \frac{5}{r}+\beta+\frac{5}{\delta_{1}^{\prime}}<2+\frac{5}{\delta}<3+\beta+\frac{5}{\delta_{1}^{\prime}} \\
& \frac{5}{r}+\frac{5}{\delta_{2}}<2+\frac{5}{\delta}<3+\frac{5}{\delta_{2}} \\
& \frac{5}{2}<\delta_{5}^{\prime}<\frac{5}{5 / \delta-(1+\beta)} \\
& \frac{5}{1+\beta}<\delta_{5}<\frac{5}{5 / \delta-2} \\
& \frac{5}{2+\beta}<\delta_{6}<\frac{5}{5 / \delta-1} \\
& 5<\delta_{6}^{\prime}<\frac{5}{5 / \delta-(2+\beta)}
\end{aligned}
$$

here (5.4) comes from (4.59), (4.64) by using the fact that $1 / \delta_{i}+1 / \delta_{i}^{\prime}=1 / \delta$, $i=1, \ldots, 6$. Moreover, $5 / \delta<3+\beta, 3 / \delta<2+\beta$. Therefore, Lemmas 4.6 and 4.7 imply

$$
\Phi: \mathfrak{M}\left(\Omega^{T}\right) \times[0,1] \rightarrow W_{\delta}^{2+\beta, 1+\beta / 2}\left(\Omega^{T}\right)
$$


LEMmA 5.1. Assume that $g^{(i)} \in W_{\delta}^{\beta, \beta / 2}\left(\Omega^{T}\right), h^{(i)}(0) \in W_{\delta}^{2+\beta-2 / \delta}(\Omega)$, $i=1,2, F^{\prime} \in W_{\delta}^{1+\beta-1 / \delta, 1 / 2+1 /(2 \beta)-1 /(2 \delta)}\left(S_{2}^{T}\right), f \in L_{6 / 5, \infty}\left(\Omega^{T}\right) \cap L_{27 / 16}\left(\Omega^{T}\right)$, $F_{3} \in L_{18 / 13}\left(\Omega^{T}\right), v(0) \in W_{27 / 16}^{22 / 27}(\Omega), \chi(0) \in L_{2}(\Omega)$. Assume that $\delta \in(1,2)$ and $\beta \in(0,1)$ are such that $5 / \delta<3+\beta, 3 / \delta<2+\beta$. Then the imbedding

$$
W_{\delta}^{2+\beta, 1+\beta / 2}\left(\Omega^{T}\right) \subset \mathfrak{M}\left(\Omega^{T}\right)
$$

is compact.

Proof. In view of the interpolation inequalities (4.57) and (4.63) for $h^{(1)}$ and $h^{(2)}$, we only have to show that

$$
W_{\delta}^{2+\beta, 1+\beta / 2}\left(\Omega^{T}\right) \subset \mathfrak{M}_{0}\left(\Omega^{T}\right)
$$

is compact.

To show this we recall that the following imbeddings are compact:

$$
\begin{array}{lll}
W_{\delta}^{2+\beta, 1+\beta / 2}\left(\Omega^{T}\right) \subset L_{\infty, 1}\left(\Omega^{T}\right) & \text { if } & \frac{3}{\delta}<2+\beta, \\
W_{\delta}^{2+\beta, 1+\beta / 2}\left(\Omega^{T}\right) \subset L_{3, \infty}\left(\Omega^{T}\right) & \text { if } & \frac{5}{\delta}<3+\beta, \\
W_{\delta}^{2+\beta, 1+\beta / 2}\left(\Omega^{T}\right) \subset L_{54 / 17}\left(\Omega^{T}\right) & \text { if } & 5\left(\frac{1}{\delta}-\frac{17}{54}\right)<2+\beta .
\end{array}
$$

This implies that (5.7) is also compact. This ends the proof.

Now we find an estimate for a fixed point of mapping (5.3).

Lemma 5.2. Assume that $g^{(i)} \in W_{\delta}^{\beta, \beta / 2}\left(\Omega^{T}\right), h^{(i)} \in W_{\delta}^{2+\beta-2 / \delta}(\Omega), i=$ $1,2, F^{\prime} \in W_{\delta}^{1+\beta-1 / \delta, 1 / 2+\beta / 2-1 /(2 \delta)}\left(S_{2}^{T}\right), F_{3} \in L_{18 / 13}\left(\Omega^{T}\right), f \in L_{6 / 5, \infty}\left(\Omega^{T}\right)$ $\cap L_{27 / 16}\left(\Omega^{T}\right), v(0) \in W_{27 / 16}^{22 / 27}(\Omega), \chi(0) \in L_{2}(\Omega)$. Assume that $\delta \in(1,2)$, $\beta \in(0,1), 5 / \delta<3+\beta, 3 / \delta<2+\beta$. Set

$$
d(T)=\sum_{i=1}^{2}\left(\left\|g^{(i)}\right\|_{2, \Omega^{T}}+\left\|h^{(i)}(0)\right\|_{2, \Omega}\right)+\left|f_{3}\right|_{2, S_{2}^{T}}+\left|F^{\prime}\right|_{2, S_{2}^{T}} .
$$

Then there exists a large constant $A$ (see (5.14) and inequality (5.13) below) such that for sufficiently small $d(T)$ the following estimate holds:

$$
\gamma(T) \equiv \sum_{i=1}^{2}\left(\left\|h^{(i)}\right\|_{2+\beta, \delta, \Omega^{T}}+\left\|\nabla q^{(i)}\right\|_{\beta, \delta, \Omega^{T}}\right) \leq A .
$$

Proof. In view of the imbeddings (5.8) we obtain from (4.49) the estimate

$$
\|v\|_{2,27 / 16, \Omega^{T}} \leq \varphi_{2}\left(\gamma(T), G_{1}(T), G_{0}(0), T\right) .
$$

Let us introduce the quantity 


$$
\begin{aligned}
D(T)= & \left\|g^{(1)}\right\|_{\beta, \delta, \Omega^{T}}+\left\|g^{(2)}\right\|_{\beta, \delta, \Omega^{T}}+\left\|F^{\prime}\right\|_{1+\beta-2 / \delta, \delta, S_{2}^{T}} \\
& +\left\|h^{(1)}(0)\right\|_{2+\beta-2 / \delta, \delta, \Omega}+\left\|h^{(2)}(0)\right\|_{2+\beta-2 / \delta, \delta, \Omega} .
\end{aligned}
$$

From (4.52), (4.66) and (5.10) we have

$$
\gamma(T) \leq \varphi\left(\gamma(T), G_{1}(T), G_{0}(0), T\right) d(T)+c_{1} D(T),
$$

where $\varphi$ is an increasing positive function.

From (5.12) it follows that for a given $T$ and sufficiently small $d(T)$ there exists a large constant $A$ such that

$$
\varphi\left(A, G_{1}(T), G_{0}(0), T\right) d(T)+c_{1} D(T) \leq A
$$

and

$$
c_{1} D(T) \leq A .
$$

Inequality (5.13) implies that

$$
\gamma(T) \leq A .
$$

Hence (5.9) holds, which ends the proof.

REMARK 5.3. Inequalities (5.12) and (5.14) imply (5.9) for $T$ such that

$$
T \leq \frac{1}{\varphi_{3}(d(T))}
$$

where $\varphi_{3}$ an increasing positive function such that $\lim _{d \rightarrow 0} \varphi_{3}(d)=0$.

Finally, we show the uniform continuity of the mapping $\Phi$ defined by (5.3).

Lemma 5.4. Let the assumptions of Lemma 5.2 hold. Then the mapping $\Phi$ is uniformly continuous in the product $\mathfrak{M}\left(\Omega^{T}\right) \times[0,1]$, where $\mathfrak{M}\left(\Omega^{T}\right)$ is defined by (5.4), (5.4').

Proof. Uniform continuity with respect to $\lambda \in[0,1]$ is evident. Therefore we examine the uniform continuity with respect to elements of $\mathfrak{M}\left(\Omega^{T}\right)$ for any $\lambda \in[0,1]$. Since dependence on $\lambda$ is very simple we omit $\lambda$ in the considerations below because it does not affect the proof.

Let $\widetilde{h}_{s}^{(i)} \in \mathfrak{M}\left(\Omega^{T}\right), s=1,2, i=1,2$, be two elements. We consider the following problems:

$$
\begin{array}{ll}
h_{s, t}^{(1)}-\operatorname{div} \mathbb{T}\left(h_{s}^{(1)}, q_{s}^{(1)}\right)=-v_{s} \cdot \nabla \widetilde{h}_{s}^{(1)}-\widetilde{h}_{s}^{(1)} \cdot \nabla v_{s}+g^{(1)} & \text { in } \Omega^{T}, \\
\operatorname{div} h_{s}^{(1)}=0 & \text { in } \Omega^{T}, \\
h_{s}^{(1)} \cdot \bar{n}=0, \quad \bar{n} \cdot \mathbb{D}\left(h_{s}^{(1)}\right) \cdot \bar{\tau}_{\alpha}=0, \quad \alpha=1,2, & \text { on } S_{1}^{T}, \\
h_{s i}^{(1)}=0, \quad i=1,2, \quad h_{s 3, x_{3}}^{(1)}=0 & \text { on } S_{2}^{T}, \\
\left.h_{s}^{(1)}\right|_{t=0}=h^{(1)}(0) & \text { in } \Omega, \\
s=1
\end{array}
$$

where $s=1,2$; 


$$
\begin{aligned}
& \chi_{s, t}+v_{s} \cdot \nabla \chi_{s}-\widetilde{h}_{s 3}^{(1)} \chi_{s}+\widetilde{h}_{s 2}^{(1)} w_{s, x_{1}} \\
& -\widetilde{h}_{s 1}^{(1)} w_{s, x_{2}}-\nu \Delta \chi_{s}=F_{3} \quad \text { in } \Omega^{T}, \\
& \chi_{s}=\sum_{i=1}^{2} v_{s i} a_{i} \equiv \chi_{s *} \quad \text { on } S_{1}^{T}, \\
& \chi_{s}=0 \quad \text { on } S_{2}^{T}, \\
& \left.\chi_{s}\right|_{t=0}=\chi(0) \quad \text { on } \Omega,
\end{aligned}
$$

where $s=1,2, a_{i}, i=1,2$, depend on $S_{1}$ and are defined by $(3.8)_{2}$;

$$
\begin{array}{ll}
v_{s 2, x_{1}}-v_{s 1, x_{2}}=\chi_{s} & \text { in } \Omega^{\prime}, \\
v_{s 1, x_{1}}+v_{s 2, x_{2}}=-h_{s 3}^{(1)} & \text { in } \Omega^{\prime}, \\
v_{s}^{\prime} \cdot \bar{n}^{\prime}=0 & \text { on } S_{1}^{\prime},
\end{array}
$$

where $s=1,2, \Omega^{\prime}$ nad $S_{1}^{\prime}$ are cross-sections of $\Omega$ and $S_{1}$ with a plane perpendicular to the $x_{3}$ axis;

$$
\begin{aligned}
& h_{s, t}^{(2)}-\operatorname{div} \mathbb{T}\left(h_{s}^{(2)}, q_{s}^{(2)}\right)=-\widetilde{h}_{s}^{(2)} \cdot \nabla v_{s}-2 \widetilde{h}_{s}^{(1)} \cdot \nabla \widetilde{h}_{s}^{(1)} \\
& -v_{s} \cdot \nabla \widetilde{h}_{s}^{(2)}+g^{(2)} \quad \text { in } \Omega^{T}, \\
& \operatorname{div} h_{s}^{(2)}=0 \\
& \text { in } \Omega^{T} \text {, } \\
& \bar{n} \cdot h_{s}^{(2)}=0, \quad \bar{n} \cdot \mathbb{D}\left(h_{s}^{(2)}\right) \cdot \bar{\tau}_{\alpha}=0, \quad \alpha=1,2, \\
& \text { on } S_{1}^{T} \text {, } \\
& h_{s 3}^{(2)}=0, \quad h_{s i, x_{3}}^{(2)}=\bar{F}_{i}, \quad i=1,2 \text {, } \\
& \text { on } S_{2}^{T} \text {, } \\
& \left.h_{s}^{(2)}\right|_{t=0}=h^{(2)}(0) \\
& \text { on } \Omega \text {, }
\end{aligned}
$$

where $s=1,2$.

First we examine problem (5.18). Let us introduce a function $\widetilde{\chi}_{s}$ as a solution to the problem

$$
\begin{array}{ll}
\tilde{\chi}_{s, t}-\nu \Delta \widetilde{\chi}_{s}=0 & \text { in } \Omega^{T}, \\
\tilde{\chi}_{s}=\chi_{s *} & \text { on } S_{1}^{T}, \\
\tilde{\chi}_{s, x_{3}}=0 & \text { on } S_{2}^{T}, \\
\left.\tilde{\chi}_{s}\right|_{t=0}=0 & \text { in } \Omega,
\end{array}
$$

where $s=1,2$. Introducing the new function

$$
\chi_{s}^{\prime}=\chi_{s}-\tilde{\chi}_{s}, \quad s=1,2,
$$

we see that it is a solution to the problem

$$
\begin{array}{ll}
\chi_{s, t}^{\prime}+v_{s} \cdot \nabla \chi_{s}^{\prime}-\widetilde{h}_{s 3}^{(1)} \chi_{s}^{\prime}+\widetilde{h}_{s 2}^{(1)} w_{s, x_{1}}-\widetilde{h}_{s 1}^{(1)} w_{s, x_{2}} & \\
\quad-\nu \Delta \chi_{s}^{\prime}=F_{3}-v_{s} \cdot \nabla \widetilde{\chi}_{s}+\widetilde{h}_{s 3}^{(1)} \widetilde{\chi}_{s} & \text { in } \Omega^{T}, \\
\chi_{s}^{\prime}=0 & \text { on } S_{1}^{T}, \\
\chi_{s, x_{3}}^{\prime}=0 & \text { on } S_{2}^{T}, \\
\left.\chi_{s}^{\prime}\right|_{t=0}=\chi_{s}(0) & \text { in } \Omega .
\end{array}
$$


Since we are looking for a solution which is a regularization of a weak solution, we need an energy type estimate for the weak solution,

$$
|v|_{2, \infty, \Omega^{t}}+|\nabla v|_{2, \Omega^{t}} \leq d_{2}(t), \quad t \leq T .
$$

Repeating the considerations leading to (4.49) we obtain

$$
\left\|v_{s}\right\|_{2,27 / 16, \Omega^{t}} \leq \varphi_{1}\left(d_{2}, \widetilde{\gamma}_{1 s}(t), G_{1}(t), G_{0}(0)\right), \quad s=1,2, t \leq T,
$$

where $\varphi_{1}$ is an increasing positive function and $\widetilde{\gamma}_{1 s}(t)$ is equal to $\gamma_{1}(t)$, where $h^{(1)}, h^{(2)}$ are replaced by $\widetilde{h}_{s}^{(1)}, \widetilde{h}_{s}^{(2)}$, respectively.

For solutions of problem (5.17) we have

$$
\begin{aligned}
\left\|h_{s}^{(1)}\right\|_{2+\beta, \delta, \Omega^{t}}+\left\|\nabla q_{s}^{(1)}\right\|_{\beta, \delta, \Omega^{t}} \leq c\left(\left\|v_{s} \cdot \nabla \widetilde{h}_{s}^{(1)}\right\|_{\beta, \delta, \Omega^{t}}\right. \\
\left.+\left\|\widetilde{h}_{s}^{(1)} \cdot \nabla v_{s}\right\|_{\beta, \delta, \Omega^{t}}+\left\|g^{(1)}\right\|_{\beta, \delta, \Omega^{t}}+\left\|h^{(1)}(0)\right\|_{2+\beta-2 / \delta, \delta, \Omega}\right) .
\end{aligned}
$$

Similarly, for solutions of problem (5.20) we get

$$
\begin{aligned}
&\left\|h_{s}^{(2)}\right\|_{2+\beta, \delta, \Omega^{t}}+\left\|\nabla q_{s}^{(2)}\right\|_{\beta, \delta, \Omega^{t}} \\
& \leq c\left(\left\|\widetilde{h}_{s}^{(2)} \cdot \nabla v_{s}\right\|_{\beta, \delta, \Omega^{t}}+\left\|\widetilde{h}_{s}^{(1)} \cdot \nabla \widetilde{h}_{s}^{(1)}\right\|_{\beta, \delta, \Omega^{t}}\right. \\
&\left.\quad+\left\|v_{s} \cdot \nabla \widetilde{h}_{s}^{(2)}\right\|_{\beta, \delta, \Omega^{t}}+\left\|g^{(2)}\right\|_{\beta, \delta, \Omega^{t}}+\left\|h^{(2)}(0)\right\|_{2+\beta-2 / \delta, \delta, \Omega}\right) .
\end{aligned}
$$

In view of the definition of the space $\mathfrak{M}_{1}\left(\Omega^{T}\right)$ and imbedding theorems, we obtain from (5.26) and (5.27) the inequalities

$$
\begin{aligned}
\left\|h_{s}^{(1)}\right\|_{2+\beta, \delta, \Omega^{t}}+\left\|\nabla q_{s}^{(1)}\right\|_{\beta, \delta, \Omega^{t}} \leq c\left\|v_{s}\right\|_{2,27 / 16, \Omega^{t}}\left\|\widetilde{h}_{s}^{(1)}\right\|_{\mathfrak{M}_{1}\left(\Omega^{t}\right)} \\
+c\left(\left\|g^{(1)}\right\|_{\beta, \delta, \Omega^{t}}+\left\|h^{(1)}(0)\right\|_{2+\beta-2 / \delta, \delta, \Omega}\right)
\end{aligned}
$$

and

$$
\begin{aligned}
& \left\|h_{s}^{(2)}\right\|_{2+\beta, \delta, \Omega^{t}}+\left\|\nabla q_{s}^{(2)}\right\|_{\beta, \delta, \Omega^{t}} \leq c\left\|v_{s}\right\|_{2,27 / 16, \Omega^{t}}\left\|\widetilde{h}_{s}^{(2)}\right\|_{\mathfrak{M}_{1}\left(\Omega^{t}\right)} \\
& +c\left\|\widetilde{h}_{s}^{(1)}\right\|_{\mathfrak{M}_{1}\left(\Omega^{t}\right)}^{2}+c\left(\left\|g^{(2)}\right\|_{\beta, \delta, \Omega^{t}}+\left\|h^{(2)}(0)\right\|_{2+\beta-2 / \delta, \delta, \Omega}\right) .
\end{aligned}
$$

In view of the definition of $\mathfrak{M}_{0}\left(\Omega^{T}\right)$ we can replace $(5.25)$ by

$$
\left\|v_{s}\right\|_{2,27 / 16, \Omega^{t}} \leq \varphi\left(\left\|\widetilde{h}_{s}^{(1)}, \widetilde{h}_{s}^{(2)}\right\|_{\mathfrak{M}_{0}\left(\Omega^{t}\right)}\right)
$$

where

$$
\|u, v\|_{\mathfrak{M}\left(\Omega^{t}\right)}=\|u\|_{\mathfrak{M}\left(\Omega^{t}\right)}+\|v\|_{\mathfrak{M}\left(\Omega^{t}\right)}
$$

From (5.28)-(5.30) we obtain

$$
\begin{aligned}
\left\|h_{s}^{(1)}, h_{s}^{(2)}\right\|_{\mathfrak{M}\left(\Omega^{t}\right)} \leq & \varphi\left(\left\|\widetilde{h}_{s}^{(1)}, \widetilde{h}_{s}^{(2)}\right\|_{\mathfrak{M}\left(\Omega^{t}\right)}\right) \\
& +c \sum_{i=1}^{2}\left(\left\|g^{(i)}\right\|_{\beta, \delta, \Omega^{t}}+\left\|h^{(i)}(0)\right\|_{2+\beta-2 / \delta, \delta, \Omega}\right),
\end{aligned}
$$

where $s=1,2, t \leq T$. Hence the transformation $\Phi$ maps bounded sets in $\mathfrak{M}\left(\Omega^{T}\right)$ into bounded sets in $\mathfrak{M}\left(\Omega^{T}\right)$. 
Now we shall show the uniform continuity of $\Phi$. For this purpose we introduce

$$
\begin{array}{ll}
H^{(i)}=h_{1}^{(i)}-h_{2}^{(i)}, & V=v_{1}-v_{2}, \\
Q^{(i)}=q_{1}^{(i)}-q_{2}^{(i)}, & K=\chi_{1}-\chi_{2}, \quad i=1,2 .
\end{array}
$$

Then the problem for $H^{(1)}$ takes the form

$$
\begin{aligned}
& H_{, t}^{(1)}-\operatorname{div} \mathbb{T}\left(H^{(1)}, Q^{(1)}\right)=-V \cdot \nabla \widetilde{h}_{1}^{(1)}-v_{2} \cdot \nabla \widetilde{H}^{(1)} \\
& -\widetilde{H}^{(1)} \cdot \nabla v_{1}-\widetilde{h}_{2}^{(1)} \cdot \nabla V \quad \text { in } \Omega^{T}, \\
& \operatorname{div} H^{(1)}=0 \quad \text { in } \Omega^{T}, \\
& H^{(1)} \cdot \bar{n}=0, \quad \bar{n} \cdot \mathbb{D}\left(H^{(1)}\right) \cdot \bar{\tau}_{\alpha}=0, \quad \alpha=1,2, \quad \text { on } S_{1}^{T}, \\
& H_{i}^{(1)}=0, \quad i=1,2, \quad H_{3, x_{3}}^{(1)}=0 \quad \text { on } S_{2}^{T}, \\
& \left.H^{(1)}\right|_{t=0}=0 \quad \text { in } \Omega \text {. }
\end{aligned}
$$

Next, we have the problem for $H^{(2)}$ :

$$
\begin{array}{ll}
H_{, t}^{(2)}-\operatorname{div} \mathbb{T}\left(H^{(2)}, Q^{(2)}\right)=-\widetilde{H}^{(2)} \cdot \nabla v_{1}-\widetilde{h}_{2}^{(2)} \cdot \nabla V & \\
-2 \widetilde{H}^{(1)} \cdot \nabla \widetilde{h}_{1}^{(1)}-2 \widetilde{h}_{2}^{(1)} \cdot \nabla \widetilde{H}^{(1)}-V \cdot \nabla \widetilde{h}_{1}^{(2)}-v_{2} \cdot \nabla \widetilde{H}^{(2)} & \text { in } \Omega^{T}, \\
\operatorname{div} H^{(2)}=0 & \text { in } \Omega^{T}, \\
\bar{n} \cdot H^{(2)}=0, \quad \bar{n} \cdot \mathbb{D}\left(H^{(2)}\right) \cdot \bar{\tau}_{\alpha}=0, \quad \alpha=1,2, & \text { on } S_{1}^{T}, \\
H_{3}^{(2)}=0, \quad H_{i, x_{3}}^{(2)}=0, \quad i=1,2, & \text { on } S_{2}^{T}, \\
\left.H^{(2)}\right|_{t=0}=0 & \text { in } \Omega .
\end{array}
$$

For solutions of (5.33) we have

$$
\begin{aligned}
\left\|H^{(1)}\right\|_{2+\beta, \delta, \Omega^{t}}+\| & \nabla Q^{(1)} \|_{\beta, \delta, \Omega^{t}} \\
\leq & c\left(\left\|V \cdot \nabla \widetilde{h}_{1}^{(1)}\right\|_{\beta, \delta, \Omega^{t}}+\left\|v_{2} \cdot \nabla \widetilde{H}^{(1)}\right\|_{\beta, \delta, \Omega^{t}}\right. \\
& \left.+\left\|\widetilde{H}^{(1)} \cdot \nabla v_{1}\right\|_{\beta, \delta, \Omega^{t}}+\left\|\widetilde{h}_{2}^{(1)} \cdot \nabla V\right\|_{\beta, \delta, \Omega^{t}}\right) .
\end{aligned}
$$

Repeating the considerations from (4.54), (4.55), (4.60), (4.61) we obtain

$$
\begin{aligned}
\left\|H^{(1)}\right\|_{2+\beta, \delta, \Omega^{t}}+\left\|\nabla Q^{(1)}\right\|_{\beta, \delta, \Omega^{t}} \leq & c\left(\|V\|_{2, r, \Omega^{t}}\left\|\widetilde{h}_{1}^{(1)}, \widetilde{h}_{2}^{(1)}\right\|_{\mathfrak{M}_{1}\left(\Omega^{t}\right)}\right. \\
& \left.+\left\|v_{1}, v_{2}\right\|_{2, r, \Omega^{t}}\left\|\widetilde{H}^{(1)}\right\|_{\mathfrak{M}_{1}\left(\Omega^{t}\right)}\right)
\end{aligned}
$$

for all $r>5 / 3$, where

$$
\left\|v_{1}, v_{2}\right\|_{2, r, \Omega^{t}}=\left\|v_{1}\right\|_{2, r, \Omega^{t}}+\left\|v_{2}\right\|_{2, r, \Omega^{t}} .
$$

For solutions of problem (5.34) we get 


$$
\begin{aligned}
& \left\|H^{(2)}\right\|_{2+\beta, \delta, \Omega^{t}}+\left\|\nabla Q^{(2)}\right\|_{\beta, \delta, \Omega^{t}} \leq c\left(\left\|\widetilde{H}^{(2)} \cdot \nabla v_{1}\right\|_{\beta, \delta, \Omega^{t}}\right. \\
& \quad+\left\|\widetilde{h}_{2}^{(2)} \cdot \nabla V\right\|_{\beta, \delta, \Omega^{t}}+\left\|\widetilde{H}^{(1)} \cdot \nabla \widetilde{h}_{1}^{(1)}\right\|_{\beta, \delta, \Omega^{t}}+\left\|\widetilde{h}_{2}^{(1)} \cdot \nabla \widetilde{H}^{(1)}\right\|_{\beta, \delta, \Omega^{t}} \\
& \left.\quad+\left\|V \cdot \nabla \widetilde{h}_{1}^{(2)}\right\|_{\beta, \delta, \Omega^{t}}+\left\|v_{2} \cdot \nabla \widetilde{H}^{(2)}\right\|_{\beta, \delta, \Omega^{t}}\right) .
\end{aligned}
$$

Repeating the estimates from (4.54), (4.55), (4.60), (4.61) and (4.68) in (5.37) yields

$$
\begin{aligned}
& \left\|H^{(2)}\right\|_{2+\beta, \delta, \Omega^{t}}+\left\|\nabla Q^{(2)}\right\|_{\beta, \delta, \Omega^{t}} \leq c\left(\|V\|_{2, r, \Omega^{t}}\left\|\widetilde{h}_{2}^{(1)}, h_{2}^{(2)}\right\|_{\mathfrak{M}_{1}\left(\Omega^{t}\right)}\right. \\
& \left.\quad+\left\|\widetilde{H}^{(2)}\right\|_{\mathfrak{M}_{1}\left(\Omega^{t}\right)}\left\|v_{1}, v_{2}\right\|_{2, r, \Omega^{t}}+\left\|\widetilde{H}^{(1)}\right\|_{\mathfrak{M}_{1}\left(\Omega^{t}\right)}\left\|\widetilde{h}_{1}^{(1)}, \widetilde{h}_{2}^{(2)}\right\|_{\mathfrak{M}_{1}\left(\Omega^{t}\right)}\right)
\end{aligned}
$$

for all $r>5 / 3$.

To show the continuity of the transformation $\Phi$ we should find an estimate for $\|V\|_{2, r, \Omega^{t}}, r=27 / 16$. For this purpose we consider the problem

$$
\begin{aligned}
& V_{, t}-\operatorname{div} \mathbb{T}(V, Q)=-V^{\prime} \cdot \nabla v_{1}-v_{2}^{\prime} \cdot \nabla V \\
& -W h_{1}^{(1)}-w_{2} H^{(1)} \quad \text { in } \Omega^{T}, \\
& \operatorname{div} V=0 \quad \text { in } \Omega^{T}, \\
& V \cdot \bar{n}=0, \quad \bar{n} \cdot \mathbb{T}(V, Q) \cdot \bar{\tau}_{\alpha}=0, \quad \alpha=1,2, \quad \text { on } S^{T}, \\
& \left.V\right|_{t=0}=0 \quad \text { in } \Omega \text {, }
\end{aligned}
$$

where $V^{\prime}=\left(V_{1}, V_{2}\right), W=V_{3}, v_{s}^{\prime}=\left(v_{s 1}, v_{s 2}\right), w_{s}=v_{s 3}$.

For solutions of (5.39) we have

$$
\begin{aligned}
\|V\|_{2, r, \Omega^{t}}+|\nabla Q|_{r, \Omega^{t}} \leq & c\left(\left|V^{\prime} \cdot \nabla v_{1}\right|_{r, \Omega^{t}}+\left|v_{2}^{\prime} \cdot \nabla V\right|_{r, \Omega^{t}}\right. \\
& \left.+\left|W h_{1}^{(1)}\right|_{r, \Omega^{t}}+\left|w_{2} H^{(1)}\right|_{r, \Omega^{t}}\right) .
\end{aligned}
$$

We bound the first term on the r.h.s. of (5.40) by

$$
c|V|_{5, \Omega^{t}}\left\|v_{1}\right\|_{2, r, \Omega^{t}} \equiv I_{1} \text {. }
$$

Since $r>5 / 3$ we have $5<\frac{5}{5 / r-2}$, so by interpolation we get, for all $\varepsilon_{1}>0$,

$$
I_{1} \leq \varepsilon_{1}\|V\|_{2, r, \Omega^{t}}+c\left(1 / \varepsilon_{1}\right) \varphi\left(\left\|v_{1}\right\|_{2, r, \Omega^{t}}\right)|V|_{2, \Omega^{t}} .
$$

Similarly, we estimate the second term on the r.h.s. of (5.40) by

$$
c|\nabla V|_{5 / 2, \Omega^{t}}\left\|v_{2}\right\|_{2, r, \Omega^{t}} \equiv I_{2} .
$$

For $r>5 / 3$ we have $\frac{5}{2}<\frac{5}{5 / r-1}$, so by interpolation, for all $\varepsilon_{2}>0$,

$$
I_{2} \leq \varepsilon_{2}\|V\|_{2, r, \Omega^{t}}+c\left(1 / \varepsilon_{2}\right) \varphi\left(\left\|v_{2}\right\|_{2, r, \Omega^{t}}\right)|V|_{2, \Omega^{t}} .
$$

By the Hölder inequality the third term on the r.h.s. of (5.40) is bounded by

$$
c|W|_{\sigma_{1}, \Omega^{t}}\left|h_{1}^{(1)}\right|_{\sigma_{2}, \Omega^{t}} \equiv I_{3},
$$

provided $5 / r-5 / \sigma_{1}<2,5 / \delta-5 / \sigma_{2} \leq 2+\beta, 1 / \sigma_{1}+1 / \sigma_{2}=1 / r$, which are satisfied for $5 / \delta<3+\beta$ and $r>5 / 3$. 
Since $5 / r-5 / \sigma_{1}<2$, we apply the interpolation inequality to the first factor in $I_{3}$ to get, for all $\varepsilon_{3}>0$,

$$
I_{3} \leq \varepsilon_{3}\|V\|_{2, r, \Omega^{t}}+c\left(1 / \varepsilon_{3}\right) \varphi\left(\left\|h_{1}^{(1)}\right\|_{2+\beta, \delta, \Omega^{t}}\right)|V|_{2, \Omega^{t}} .
$$

Finally, by the Hölder inequality, the fourth term on the r.h.s. of (5.40) is estimated by

$$
c\left|w_{2}\right|_{\varrho_{1}, \Omega^{t}}\left|H^{(1)}\right|_{\varrho_{2}, \Omega^{t}} \equiv I_{4},
$$

provided $1 / \varrho_{1}+1 / \varrho_{2}=1 / r, 5 / r-5 / \varrho_{1}=2$, that is, $\varrho_{2}=5 / 2$. Hence,

$$
I_{4} \leq c\left\|v_{2}\right\|_{2, r, \Omega^{t}}\left|H^{(1)}\right|_{5 / 2, \Omega^{t}} .
$$

Applying the above estimates in (5.40) and assuming that $\varepsilon_{1}, \varepsilon_{2}, \varepsilon_{3}$ are sufficiently small we obtain

$$
\begin{aligned}
& \|V\|_{2, r, \Omega^{t}}+|\nabla Q|_{r, \Omega^{t}} \\
& \quad \leq \varphi\left(\left\|v_{1}, v_{2}\right\|_{2, r, \Omega^{t}},\left\|h_{1}^{(1)}\right\|_{2+\beta, \delta, \Omega^{t}}\right) \cdot\left(|V|_{2, \Omega^{t}}+\left|H^{(1)}\right|_{5 / 2, \Omega^{t}}\right),
\end{aligned}
$$

provided $r>5 / 3,5 / \delta<3+\beta$.

Assume that $\widetilde{h}_{s}^{(1)}, \widetilde{h}_{s}^{(2)}, s=1,2$, belong to a bounded set in $\mathfrak{M}\left(\Omega^{T}\right)$. Hence, there exists a constant $A$ such that

$$
\left\|\widetilde{h}_{s}^{(1)}, \widetilde{h}_{s}^{(2)}\right\|_{\mathfrak{M}\left(\Omega^{T}\right)} \leq A .
$$

Making use of (5.30), (5.31) and (5.42) in (5.41) implies

$$
\|V\|_{2, r, \Omega^{t}}+|\nabla Q|_{r, \Omega^{t}} \leq \varphi(A)\left(|V|_{2, \Omega^{t}}+\left|H^{(1)}\right|_{5 / 2, \Omega^{t}}\right) .
$$

Finally, we estimate the r.h.s. of (5.43). Multiplying $(5.33)_{1}$ by $H^{(1)}$ and integrating over $\Omega$ yields

$$
\begin{aligned}
\frac{d}{d t}\left|H^{(1)}\right|_{2, \Omega}^{2}+\nu\left\|H^{(1)}\right\|_{1, \Omega}^{2} \leq & c\left(\left|V \cdot \nabla \widetilde{h}^{(1)}\right|_{6 / 5, \Omega}^{2}+\left|v_{2} \cdot \nabla \widetilde{H}^{(1)}\right|_{6 / 5, \Omega}^{2}\right. \\
& \left.+\left|\widetilde{H}^{(1)} \cdot \nabla v_{1}\right|_{6 / 5, \Omega}^{2}+\left|\widetilde{h}_{2}^{(1)} \cdot \nabla V\right|_{6 / 5, \Omega}^{2}\right) .
\end{aligned}
$$

By the Hölder inequality, (5.44) implies, for $\lambda_{1}, \lambda_{2}$ such that $1 / \lambda_{1}+1 / \lambda_{2}=1$,

$$
\begin{aligned}
\frac{d}{d t}\left|H^{(1)}\right|_{2, \Omega}^{2}+ & \nu\left\|H^{(1)}\right\|_{1, \Omega}^{2} \\
\leq & c\left(|V|_{2, \Omega}^{2}\left|\nabla \widetilde{h}^{(1)}\right|_{3, \Omega}^{2}+\left|v_{2}\right|_{(6 / 5) \lambda_{1}, \Omega}^{2}\left|\nabla \widetilde{H}^{(1)}\right|_{(6 / 5) \lambda_{2}, \Omega}^{2}\right. \\
& \left.+\left|\widetilde{H}^{(1)}\right|_{2, \Omega}^{2}\left|\nabla v_{1}\right|_{3, \Omega}^{2}+\sup _{t}\left|\widetilde{h}_{2}^{(1)}\right|_{3, \Omega}^{2}|\nabla V|_{2, \Omega}^{2}\right),
\end{aligned}
$$

where, in view of (5.42), the first and last expressions are estimated by $\varphi(A)\|V\|_{1, \Omega}^{2}$.

Multiplying (5.39) $)_{1}$ by $V$ and integrating over $\Omega$ shows that

$$
\begin{aligned}
\frac{d}{d t}|V|_{2, \Omega}^{2}+\nu\|V\|_{1, \Omega}^{2} \leq & c\left(|V|_{2, \Omega}^{2}\left|\nabla v_{1}\right|_{3, \Omega}^{2}+|V|_{2, \Omega}^{2}\left|h_{1}^{(1)}\right|_{3, \Omega}^{2}\right. \\
& \left.+\left|w_{2}\right|_{3, \Omega}^{2}\left|H^{(1)}\right|_{2, \Omega}^{2}\right)
\end{aligned}
$$


Multiplying (5.46) by a constant $c_{*}$ such that $\nu c_{*}-c \varphi(A) \geq \nu$ and adding to $(5.45)$, we get

$$
\begin{aligned}
\frac{d}{d t}\left(c_{*}|V|_{2, \Omega}^{2}\right. & \left.+\left|H^{(1)}\right|_{2, \Omega}^{2}\right)+\nu\left(\|V\|_{1, \Omega}^{2}+\left\|H^{(1)}\right\|_{1, \Omega}^{2}\right) \\
\leq & c\left(\left|\widetilde{H}^{(1)}\right|_{2, \Omega}^{2}\left|\nabla v_{1}\right|_{3, \Omega}^{2}+|V|_{2, \Omega}^{2}\left|\nabla v_{1}\right|_{3, \Omega}^{2}+|V|_{2, \Omega}^{2}\left|h_{1}^{(1)}\right|_{3, \Omega}^{2}\right. \\
& \left.+\left|w_{2}\right|_{3, \Omega}^{2}\left|H^{(1)}\right|_{2, \Omega}^{2}+c\left|v_{2}\right|_{(6 / 5) \lambda_{1}, \Omega}^{2}\left|\nabla \widetilde{H}^{(1)}\right|_{(6 / 5) \lambda_{2}, \Omega}^{2}\right) .
\end{aligned}
$$

Integrating (5.47) with respect to time yields

$$
|V(t)|_{2, \Omega}^{2}+\left|H^{(1)}(t)\right|_{2, \Omega}^{2}+\nu \int_{0}^{t}\left(\left\|V\left(t^{\prime}\right)\right\|_{1, \Omega}^{2}+\left\|H^{(1)}\left(t^{\prime}\right)\right\|_{1, \Omega}^{2}\right) d t^{\prime}
$$

$$
\leq c \exp \left[c\left(\left|\nabla v_{1}\right|_{3,2, \Omega^{t}}^{2}+\left|h^{(1)}\right|_{3,2, \Omega^{t}}^{2}+|v|_{3,2, \Omega^{t}}^{2}\right)\right]
$$

$$
\cdot\left(\left|v_{2}\right|_{(6 / 5) \lambda_{1}, 2 \mu_{1}, \Omega^{t}}^{2}\left|\nabla \widetilde{H}^{(1)}\right|_{(6 / 5) \lambda_{2}, 2 \mu_{2}, \Omega^{t}}^{2}+\left|\nabla v_{1}\right|_{3,2, \Omega^{t}}^{2}\left|\widetilde{H}^{(1)}\right|_{2, \infty, \Omega^{t}}\right) \equiv J,
$$

provided $1 / \lambda_{1}+1 / \lambda_{2}=1$ and $1 / \mu_{1}+1 / \mu_{2}=1$. By imbedding we get

$$
\begin{aligned}
J \leq & c \exp \left[c\left(\left\|v_{1}\right\|_{2, r, \Omega^{t}}^{2}+\left\|h^{(1)}\right\|_{2+\beta, \delta, \Omega^{t}}^{2}\right)\right] \\
& \cdot\left(\left|v_{2}\right|_{3, \infty, \Omega^{t}}^{2}\left|\nabla \widetilde{H}^{(1)}\right|_{2, \Omega^{t}}^{2}+\left|\nabla v_{1}\right|_{3,2, \Omega^{t}}^{2}\left|\widetilde{H}^{(1)}\right|_{2, \infty, \Omega^{t}}\right) \equiv J_{1} .
\end{aligned}
$$

By (5.30), (5.31) and (5.42) we obtain

$$
J_{1} \leq \varphi(A)\left(\left|\nabla \widetilde{H}^{(1)}\right|_{2, \Omega^{t}}^{2}+\left|\widetilde{H}^{(1)}\right|_{2, \infty, \Omega^{t}}^{2}\right) .
$$

Therefore, (5.48) takes the form

$$
\|V\|_{V_{2}^{0}\left(\Omega^{t}\right)}+\left\|H^{(1)}\right\|_{V_{2}^{0}\left(\Omega^{t}\right)} \leq \varphi(A)\left(\left|\nabla \widetilde{H}^{(1)}\right|_{2, \Omega^{t}}+\left|\widetilde{H}^{(1)}\right|_{2, \infty, \Omega^{t}}\right) .
$$

Employing (5.49) in (5.43) and the result in (5.36) and (5.38) we obtain

$$
\left\|H^{(1)}, H^{(2)}\right\|_{\mathfrak{M}\left(\Omega^{T}\right)} \leq \varphi(A)\left\|\widetilde{H}^{(1)}, \widetilde{H}^{(2)}\right\|_{\mathfrak{M}\left(\Omega^{T}\right)} .
$$

This implies the uniform continuity of $\Phi$ and ends the proof.

Proof of Theorem 1. Lemmas 5.1, 5.2, 5.4 and the Leray-Schauder fixed point theorem imply that there exists a fixed point of transformation (5.3). Hence there exists a solution to problem (1.1) and Theorem 1 is proved.

Proof of Theorem 2. Assume that we have two solutions $\left(v_{i}, p_{i}\right), i=1,2$, of problem (1.1). Then $V=v_{1}-v_{2}, P=p_{1}-p_{2}$ are solutions to the problem

$$
\begin{array}{ll}
V_{, t}-\operatorname{div} \mathbb{T}(V, P)=-V \cdot \nabla v_{1}-v_{2} \cdot \nabla V & \text { in } \Omega^{T}, \\
\operatorname{div} V=0 & \text { in } \Omega^{T}, \\
V \cdot \bar{n}=0, \quad \bar{n} \cdot \mathbb{D}(V) \cdot \bar{\tau}_{\alpha}=0, \quad \alpha=1,2, & \text { on } S^{T}, \\
\left.V\right|_{t=0}=0 & \text { in } \Omega .
\end{array}
$$


Multiplying (5.51) by $V$ and integrating over $\Omega$ we obtain

$$
\frac{d}{d t}|V|_{2, \Omega}^{2}+\nu\|V\|_{1, \Omega}^{2} \leq c\left|\nabla v_{1}\right|_{3, \Omega}^{2}|V|_{2, \Omega}^{2} .
$$

For $v_{1} \in L_{2}\left(0, T ; W_{3}^{1}(\Omega)\right)$ we obtain $|V(t)|_{2, \Omega}=0$, and this ends the proof.

\section{References}

[1] W. Alame, On existence of solutions for the nonstationary Stokes system with slip boundary conditions in general Sobolev-Slobodetskii and Besov spaces, Banach Center Publ., to appear.

[2] J. D. Avrin, Large-eigenvalue global existence and regularity results for the NavierStokes equation, J. Differential Equations 127 (1996), 365-390.

[3] -, A one-point attractor theory for the Navier-Stokes equations on thin domains with no-slip boundary conditions, Proc. Amer. Math. Soc. 127 (1999), 725-735.

[4] A. Babin, A. Mahalov and B. Nicolaenko, 3D Navier-Stokes and Euler equations with initial data characterized by uniformly large vorticity, Indiana Univ. Math. J. 50 (2001), 1-35.

[5] - , - - Global regularity of $3 D$ rotating Navier-Stokes equations for resonant domains, ibid. 48 (1999), 1133-1176.

[6] O. V. Besov, V. P. Il'in and S. M. Nikol'skiŭ, Integral Representations of Functions and Imbedding Theorems, Nauka, Moscow, 1975 (in Russian).

[7] D. Iftimie and G. Raugel, Some results on the Navier-Stokes equations in thin $3 D$ domains, J. Differential Equations 169 (2001), 281-331.

[8] T. Kobayashi and W. M. Zajączkowski, On global motion of a compressible barotropic viscous fluid with boundary slip condition, Appl. Math. (Warsaw) 26 (1999), 159-194.

[9] O. A. Ladyzhenskaya, Mathematical Theory of Viscous Incompressible Fluid, Nauka, Moscow, 1970 (in Russian).

[10] - Solutions "in the large" of the nonstationary boundary value problem for the Navier-Stokes system with two space variables, Comm. Pure Appl. Math. 12 (1959), 427-433.

[11] -, On unique global solvability of the three-dimensional Cauchy problem for the Navier-Stokes equations in the presence of axial symmetry, Zap. Nauchn. Sem. LOMI 7 (1968), 155-177 (in Russian).

[12] A. Mahalov, E. S. Titi and S. Leibovich, Invariant helical subspaces for the NavierStokes equations, Arch. Rat. Mech. Anal. 112 (1990), 193-222.

[13] G. Raugel and G. R. Sell, Navier-Stokes equations on thin 3D domains. I: Global attractors and global regularity of solutions, J. Amer. Math. Soc. 6 (1993), 503-568.

[14] -, 一, Navier-Stokes equations on thin 3D domains. II: Global regularity on spatially periodic solutions, in: Nonlinear Partial Differential Equations and Their Applications), Collège de France Seminar, Vol XI, H. Brezis and J.-L. Lions (eds.), Pitman Res. Notes Math. Ser. 299, Longman Sci. Tech., 1994, 205-247.

[15] - , - Navier-Stokes equations on thin 3D domains. III: Existence of a global and local attractor, in: Turbulence in Fluid Flows, IMA Vol. Math. Appl. 55, G. R. Sell et al. (eds.), Springer, New York, 1993, 137-163. 
[16] V. A. Solonnikov and V. E. Shchadilov, On a boundary value problem for a stationary system of the Navier-Stokes equations, Trudy Mat. Inst. Steklov. 125 (1973), 196-210 (in Russian); English transl.: Proc. Steklov Inst. Math. 125 (1973), 186-199.

[17] M. R. Ukhovskiı̆ and V. I. Yudovich, Axially symmetric motions of ideal and viscous fluids filling all space, Prikl. Mat. Mekh. 32 (1968), 59-69 (in Russian).

[18] W. M. Zajacczkowski, Global special regular solutions to the Navier-Stokes equations in a cylindrical domain under boundary slip conditions, in: Gakuto Series in Math. 21 (2004), 1-188.

[19] —, Global special regular solutions to the Navier-Stokes equations in a cylindrical domain without the axis of symmetry, Topol. Methods Nonlinear Anal. 24 (2004), 69-105.

[20] —, Global existence of axially symmetric solutions of incompressible Navier-Stokes equations with large angular component of velocity, Colloq. Math. 100 (2004), 243263.

[21] — Global special regular solutions to the Navier-Stokes equations in axially symmetric domains under boundary slip conditions, Dissertationes Math., to appear.

Institute of Mathematics

Polish Academy of Sciences

Śniadeckich 8

00-956 Warszawa, Poland

E-mail:wz@impan.gov.pl
Institute of Mathematics and Cryptology

Military University of Technology

Kaliskiego 2

00-908 Warszawa, Poland

Received May 21, 2004

Revised version January 26, 2005 